Nuclear Engineering and Design 52 (1979) 385-409

(c) North-Holland Publishing Company

\title{
ON THREE-DIMENSIONAL NONLINEAR ANALYSIS OF CONCRETE STRUCTURES
}

\author{
Klaus-Jürgen BATHE and Seshadri RAMASWAMY \\ Massachusetts Institute of Technology, Cambridge, MA 02139, USA
}

Received 21 October 1978

\begin{abstract}
Solution capabilities for three-dimensional geometric and material nonlinear finite element analysis of concrete structures are presented. The concrete material is modeled including triaxial nonlinear stress-strain behavior, tensile cracking, compression crushing and strain-softening. The objective in this work was the development of a practical nonlinear concrete analysis capability. The material model can also be employed to represent some rock materials. The results of various sample analyses are given, in which the stability and accuracy of the finite element representations have been studied.
\end{abstract}

\section{Introduction}

During recent years interest in nonlinear analysis of concrete structures has increased steadily, because of the wide use of plain, reinforced and prestressed concrete as a structural material, and because of the development of relatively powerful analysis techniques implemented on electronic digital computers. A most important analysis procedure that is already in wide use for the linear analysis of structures is the finite element method $[1,2]$. If a realistic nonlinear analysis of a concrete structure can be carried out, the safety of the structure is increased and the cost can frequently be reduced.

Concrete exhibits a complex structural response with various important nonlinearities; namely, a nonlinear stress-strain behavior, tensile cracking and compression crushing material failures, and temperature dependent creep strains [3-16]. All these concrete nonlinearities depend strongly on the triaxial state of stress, and in addition the nonlinearities introduced by the reinforcing and prestressing steel should in general be taken into account

There are a number of factors that prevent at present the wide acceptability of nonlinear finite element analysis procedures in the analysis of concrete structures. A first important consideration is that the constitutive properties of concrete have not as yet been identified completely, and there is at present no generally accepted material law available to model concrete behavior. A second important factor is that nonlinear finite element analysis of concrete structures is very costly and requires much user sophistication. The high cost of nonlinear analysis of concrete structures is largely due to the difficulties encountered in the stability and accuracy of the solutions. These difficulties, however, are a direct consequence of the specific numerical implementation of the concrete nonlinearities. Since even linear three-dimensional analysis can be expensive to the analyst, the practical difficulties of three-dimensional nonlinear concrete analysis are particularly pronounced.

It is important to realize that progress in practical nonlinear analysis procedures is largely based on the development of improved constitutive models and kinematic descriptions, and on the development of stable and effective computational procedures. Since there is a strong interaction between the development of improved constitutive models and their effective numerical implementation, it is important to endeavor to advance the development of new material descriptions and their numerical implementations at the same time. The situation at present is that a linear analysis of a concrete structure can be performed in almost a routine manner, but nonlinear analyses that would represent the structural behavior more accurately, and that must he emninved to predict the ultimate load carrying capacity of a structure are difficult, if not impossible, to perform. Also, only a few general analysis tools are available for such a task. 
The objective in this paper is to present the formulation and numerical implementation of a three-dimensional concrete model that has been incorporated and evaluated in the computer program ADINA [17]. Based on the above considerations, the basic aim in this work was to implement in the program a model that with the present constitutive descriptions, numerical methods and computing equipment available and with the general high cost of three-dimensional analysis would satisfy the following two criteria. Firstly, the model should be as simple as possible, but reproduce the important nonlinear and strength characteristics consistent with experimental results. Secondly, the model should be theoretically sound and numerically stable, so that reliable analysis results are obtained.

The material model is a hypoelastic model based on a uniaxial stress-strain relation that is generalized to take biaxial and triaxial stress conditions into account. Tensile cracking and compression crushing conditions are identified using failure surfaces. The use of tensile and compression failure criteria (including strain-softening conditions) prevents that unrealistically large stress and strain conditions are predicted as can be the case when using some plasticity models, (e.g. Drucker-Prager model with Prandtl-Reuss plasticity theory).

The concrete model is defined with a number of input parameters that provide versatility in its use. By employing the appropriate material parameters, the model can be employed also to represent some rock materials [18].

In this paper, we first review the kinematic nonlinear incremental formulation that is employed in the analysis. Then the material model which has been implemented is described. The material representation includes triaxial nonlinear stress-strain behavior, material tensile cracking and compression crushing characteristics and strainsoftening effects. The model can be employed in two- and three-dimensional analysis. Following the general description of the model the computer implementation is presented. Finally, a few sample solutions are given. In the studies, various nonlinear characteristics of the material model have been evaluated in detail, in order to identify the stability and accuracy characteristics of the finite element representations.

\section{The governing incremental equilibrium equations}

A very general geometric and material nonlinear formulation is obtained using the principle of virtual displacements. Using this principle in the total Lagrangian formulation, the governing equilibrium equation at time $t+\Delta t$ for a body undergoing large displacements and exhibiting constitutive nonlinearities is $[19,20]$,

$$
\int_{0 \mathrm{~V}}^{t+\Delta t}{ }_{0} S_{i j} \delta^{t+\Delta t}{ }_{0} \epsilon_{i j}{ }^{0} \mathrm{~d} v={ }^{t+\Delta t} \mathcal{R}
$$

where the ${ }^{t+\Delta t}{ }_{0} S_{i j}$ are the components of the 2nd Piola-Kirchhoff stress tensor referred to the body configuration at time 0 , and the ${ }^{t+\Delta t} \epsilon_{i j}$ are the components of the Green-Lagrange strain tensor,

$$
{ }^{t+\Delta t}{ }_{0}^{t} \epsilon_{i j}=\frac{1}{2}\left({ }^{t+\Delta t}{ }_{0} u_{i, j}+{ }^{t+\Delta t}{ }_{0} u_{j, i}+{ }^{t+\Delta t}{ }_{0} u_{k, i}{ }^{t+\Delta t}{ }_{0} u_{k, j}\right), \quad{ }^{t+\Delta t}{ }_{0} u_{i, j}=\partial^{t+\Delta t} u_{i} / \partial^{0} x_{j},
$$

where the ${ }^{t+\Delta t} u_{i}, i=1,2,3$ are the displacement components at time $t+\Delta t$, and the ${ }^{0} x_{j}, j=1,2,3$, denote the coordinates of the body at time 0 . The symbol " $\delta$ " in eq. (1) means "variation in", and ${ }^{t+\Delta t} R$ is the total external virtual work that is performed by the body forces and surface tractions when the body is subjected to a variation in the displacements at time $t+\Delta t$.

In the incremental solution of eq. (1), we assume that the solution is known at time $t$. Then, linearizing eq. (1) about the state at time $t$, and using a modified Newton--Raphson iteration for the solution at time $t+\Delta t$, we obtain the governing equation (for static analysis or dynamic analysis with implicit time integration) [20]

$$
\int_{0_{\mathrm{V}}}{ }_{0} C_{i j r s} \delta{ }_{0} e_{i j} \Delta_{0} e_{r s}^{(k) 0} \mathrm{~d} v+\int_{0_{\mathrm{V}}}{ }_{0}^{t} S_{i j} \delta \Delta_{0} \eta_{i j}^{(k) 0} \mathrm{~d} v={ }^{t+\Delta t} \mathcal{R}-\int_{{ }_{0} \mathrm{~V}}{ }^{t+\Delta t}{ }_{0}^{(k-1)} S_{i j}{ }^{t+\Delta t}{ }_{0} \epsilon_{i j}^{(k-1)}{ }^{0} \mathrm{~d} v,
$$

where the ${ }_{0} C_{i i r s}$ are the components of the tangent constitutive tensor at time $t$, the ${ }_{0}^{t} S_{i j}$ are components of the 
2nd Piola-Kirchhoff stress tensor at time $t$, and

$$
\begin{aligned}
& \Delta_{0} e_{i j}^{(k)}=\frac{1}{2}\left(\Delta_{0} u_{l, j}^{(k)}+\Delta_{0} u_{f, i}^{(k)}+{ }_{0}^{t} u_{l, i} \Delta_{0} u l_{l, j}^{(k)}+{ }_{0}^{t} u_{l, j} \Delta_{0} u l_{l, i}^{(k)}\right), \quad \Delta_{0} \eta_{l j}^{(k)}=\frac{1}{2} \Delta_{0} u_{l, i} \Delta_{0} u_{l, j}, \\
& { }^{t+\Delta t} u_{i}^{(k)}={ }^{t+\Delta t} u_{l}^{(k-1)}+\Delta u_{i}^{(k)} .
\end{aligned}
$$

Also, the ${ }^{t+\Delta t}{ }_{0} S_{i j}^{(k-1)}$ and ${ }^{t+\Delta t} \epsilon_{i j}^{(k-1)}$ are components of the 2 nd Piola-Kirchhoff stress tensor and Green-Lagrange strain tensor corresponding to the displacements ${ }^{t+\Delta t} u_{i}^{(k-1)}$.

It should be noted that in eq. (3), the displacements are updated until the right-hand-side of the equation is zero. At this point, the equilibrium configuration corresponding to the loading at time $t+\Delta t$ has been established.

For the finite element solution, we are using isoparametric finite element discretization, in which at any time $t$ $[2]$,

$$
{ }^{t} x_{i}=\sum_{j=1}^{N} h_{j}{ }^{t} x_{i}^{j}, \quad{ }^{t} u_{i}=\sum_{j=1}^{N} h_{j}{ }^{t} u_{i}^{j},
$$

where ${ }^{t} x_{i}^{j}$ is the coordinate and ${ }^{t} u_{i}^{j}$ is the displacement of element nodal point $j$ at time $t$ and in direction $i ; i=1$, 2, 3 in three-dimensional analysis. Substituting the relations in eq. (5) into eq. (3) and introducing inertia forces as part of the body forces, we obtain the discretized equilibrium equations

$$
M^{t+\Delta t} \ddot{U}^{(k)}+\left({ }_{0}^{t} K_{\mathrm{L}}+{ }_{0}^{t} K_{\mathrm{NL}}\right) \Delta U^{(k)}={ }^{t+\Delta t} R-{ }^{t+\Delta t} F_{0}^{(k-1)}
$$

where $M$ is the time independent mass matrix, the ${ }_{0}^{t} K_{\mathrm{L}}$ and ${ }_{0}^{t} K_{\mathrm{NL}}$ are the linear and nonlinear strain stiffness matrices, ${ }^{t+\Delta t} R$ is the externally applied nodal point force vector, the vector $U$ lists the nodal point displacements, and ${ }^{t+\Delta t} F_{0}^{(k-1)}$ is the nodal point force vector that is work equivalent to the element stresses. Table 1 summarizes, for a single element, the calculation of the matrices ${ }_{0}^{t} K_{\mathrm{L}},{ }_{0}^{t} K_{\mathrm{NL}}$ and vector ${ }^{t+\Delta t}{ }_{0}^{t} F^{(k-1)}$ used in eq. (6).

In the finite element solution using eq. (6), we iterate, in essence, until the finite element system is in equilibrium. Since a displacement-based compatible finite element discretization is employed, the compatibility conditions are also satisfied. Hence, any errors in the solution beyond the finite element discretization errors which of course are encountered in linear analysis also, are those introduced in the inaccurate calculation of the constitutive relations. Since the stress-strain relationships depend on the stress and strain histories, it is important to integrate the stresses and strains accurately in the incremental solution and, in general [20]

$$
{ }^{t+\Delta t} S_{0}^{(k-1)}={ }_{0}^{t} S_{i j}+\int_{\delta}^{t+\Delta t \epsilon_{r s}^{(k-1)}}{ }_{0} C_{i j r s} \mathrm{~d} \epsilon_{r s},
$$

where ${ }^{t+\Delta t}{ }_{0} \epsilon_{r s}^{(k-1)}$ are the total Green-Lagrange strains corresponding to the loading at time $t+\Delta t$ and at the start of iteration $(k)$.

Eq. (6) holds for large displacements and large strains, but the appropriate constitutive relations must be defined. Concrete and rock materials cannot sustain large relative deformations and it is appropriate to assume in most analyses infinitesimal displacement conditions. In these analyses, all nonlinear strain terms are neglected, i.e., the nonlinear strain stiffness matrix ${ }_{0}^{t} K_{\mathrm{NL}}$ and the nonlinear strain contributions in ${ }^{t+\Delta t}{ }_{0}^{(k-1)}$ are not included in the solution, and the 2 nd Piola-Kirchhoff stresses ${ }_{0}^{t} S_{i j}$ reduce to the engineering physical stresses, ${ }^{t} \sigma_{i j}$. How . ever, although the material can only sustain small relative deformations, in some cases, rigid body rotations of the material may be significant. These large rotation effects are directly taken into account in the total Lagrangian formulation, because the 2nd Piola-Kirchhoff stresses are numerically equal to the rotated physical engineering stresses. Hence the constitutive relations once formulated for infinitesimal displacement conditions can directly be employed in the total Lagrangian formulation in order to account for large rotation effects. 
Table 1

Evaulation of stiffness matrix and nodal point force vector equivalent to element stresses

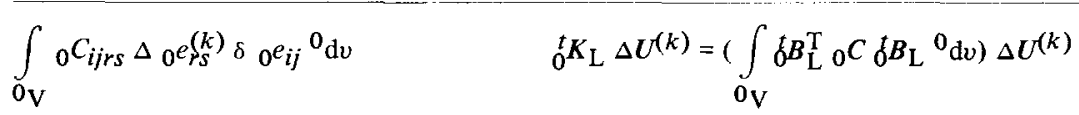

$$
\begin{aligned}
& \int_{0_{\mathrm{V}}} \delta^{t} S_{i j} \delta \Delta{ }_{0} \eta_{l j}^{(k) 0_{\mathrm{d} v}} \quad{ }_{0}^{t} K_{\mathrm{NL}} \Delta U^{(k)}=\left(\int_{0_{\mathrm{V}}}{ }_{0}^{t} B_{\mathrm{NL}}^{\mathrm{T}}{ }_{0}^{t} S_{0}^{t} B_{\mathrm{NL}}{ }^{0} \mathrm{~d} v\right) \Delta U^{(k)} \\
& \int_{0 \mathrm{~V}}{ }^{t+\Delta} \delta S_{l j}^{(k-1)} \delta{ }^{t+\Delta t} \epsilon_{i j}^{(k-1) 0_{\mathrm{d} v}} \quad{ }^{t+\Delta t} F_{0}^{(k-1)}=\int_{0_{\mathrm{V}}}{ }_{0}^{t+\Delta t} B_{\mathrm{L}}^{(k-1) \mathrm{T} t+\Delta t} \hat{0}^{(k-1) 0_{\mathrm{S}}}
\end{aligned}
$$

${ }_{0}^{t} B_{\mathrm{L}}=$ linear strain-displacement transformation matrix corresponding to time $t$.

${ }_{0}^{t} B_{\mathrm{NL}}=$ nonlinear strain-displacement transformation matrix corresponding to time $t$.

${ }_{0}^{t} S=$ stress matrix of stresses at time $t$.

${ }^{t+\Delta t} \hat{0}^{(k-1)}=$ stress vector of stresses corresponding to time $t^{\prime}+\Delta t$ and iteration $(k-1)$.

${ }_{0}^{t+\Delta t} B_{\mathrm{L}}^{(k-1)}=$ linear strain-displacement transformation matrix corresponding to time $t+\Delta t$ and iteration $(k-1)$.

\section{The concrete or rock material model}

The model implemented employs three basic features to describe the material behavior, namely, (i) a nonlinear stress-strain relation including strain-softening to allow for the weakening of the material under increasing compressive stresses, (ii) a failure envelope that defines cracking in tension and crushing in compression, and (iii) a strategy to model the post-cracking and crushing behavior of the material. In the solution, the material can be subjected to cyclic loading conditions, i.e., the numerical solution allows for unloading and reloading including deactivation of tensile failures.

In the following, the material model is described for infinitesimal displacement conditions using the engineering stresses ${ }^{t} \sigma_{i j}$ and engineering strains ${ }^{t} e_{i j}$. In order to analyze problems with large rotation conditions, the total Lagrangian stress and strain variables must be substituted for the engineering variables [20].

\subsection{Stress-strain relations}

The general multiaxial stress -strain relations are derived from a uniaxial stress-strain relation ${ }^{t} \widetilde{\sigma}$ versus ${ }^{\tau} \widetilde{e}[4-$ 7]. In this section, we describe the uniaxial and multiaxial stress-strain relations employed in the model prior to tensile cracking or compression crushing.

In the following discussion, all uniaxial parameters are identified by a curl $(\sim)$ placed over them, (i.e., all parameters that have been obtained from fig. 1 carry a curl).

\subsubsection{Uniaxial conditions}

A typical uniaxial stress ${ }^{t} \widetilde{\sigma}$ to uniaxial strain ${ }^{t} \widetilde{e}$ relation (assuming loading of the material) is shown in fig. 1. This stress-strain relation shows that there are basically three strain phases; namely, corresponding to $t_{e} \geqslant 0$, $0>{ }^{t} \tilde{e} \geqslant \widetilde{e}_{\mathrm{c}}$ and $\tilde{e}_{\mathrm{c}}>{ }^{t} \widetilde{e} \geqslant \widetilde{e}_{\mathrm{u}}$ where $\widetilde{e}_{\mathrm{c}}$ is the strain corresponding to the minimum (crushing) stress, $\widetilde{\sigma}_{\mathrm{c}}$, that can be reached, and $\tilde{e}_{\mathrm{u}}$ is the ultimate compressive strain. If ${ }^{t} \widetilde{e}>0$, i.e. the material is in tension, the stress-strain relation is linear and a constant Young's modulus, $\widetilde{E}_{0}$, is employed.

$$
\begin{aligned}
& { }^{t} \tilde{\sigma}=\widetilde{E}_{0}{ }^{t} \tilde{e}, \\
& \frac{\mathrm{d}^{t} \widetilde{\sigma}}{\mathrm{d}^{t} \widetilde{e}}=\widetilde{E}_{0} .
\end{aligned}
$$




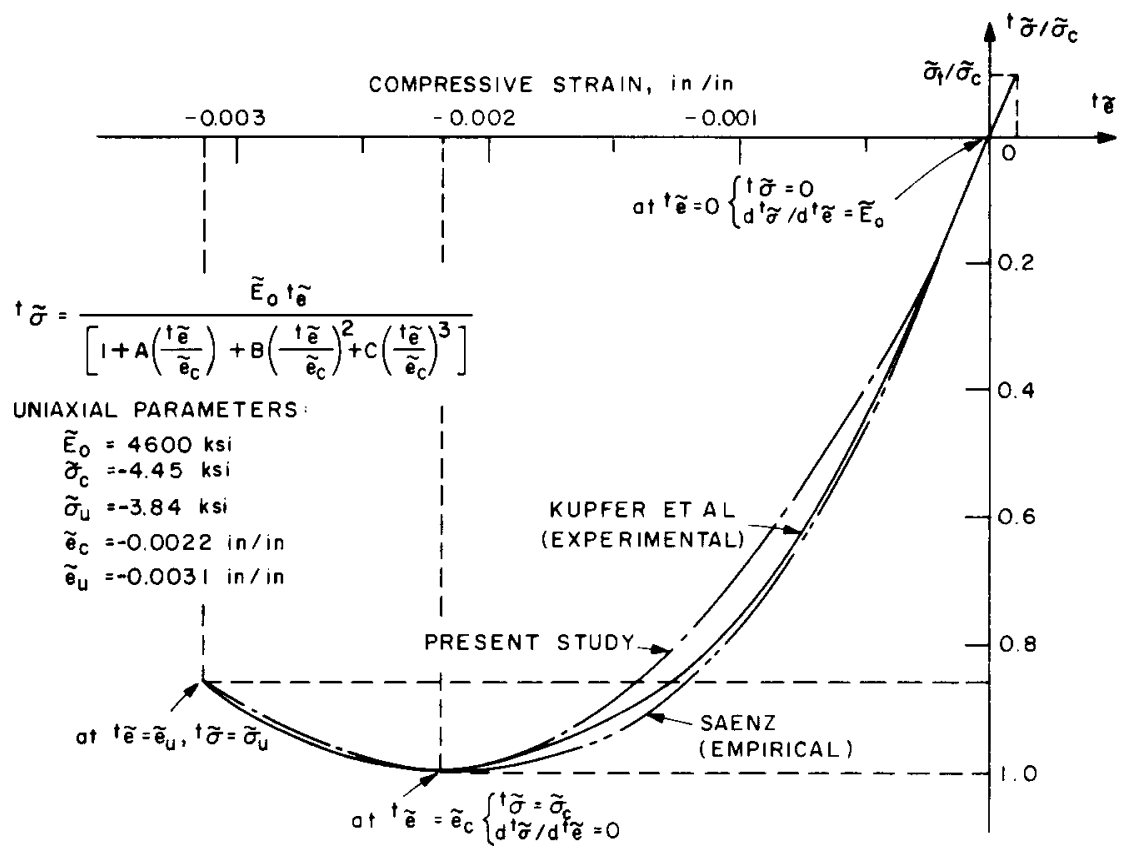

Fig. 1. Material model uniaxial stress-strain law.

For ${ }^{t} \tilde{e} \leqslant 0$, we assume the following relation,

$$
{ }^{t} \widetilde{\sigma} / \widetilde{\sigma}_{\mathrm{c}}=\frac{\left(\widetilde{E}_{0} / \widetilde{E}_{\mathrm{s}}\right)\left({ }^{t} \widetilde{e} / \widetilde{e}_{\mathrm{c}}\right)}{1+A\left({ }^{\tau} \widetilde{e} / \widetilde{e}_{\mathrm{c}}\right)+B\left({ }^{t} \widetilde{e} / \widetilde{e}_{\mathrm{c}}\right)^{2}+C\left({ }^{t} \widetilde{e} / \widetilde{e}_{\mathrm{c}}\right)^{3}},
$$

and hence,

$$
{ }^{t} \widetilde{E}=\frac{\widetilde{E}_{\mathrm{o}}\left[1-B\left({ }^{t} \widetilde{e} / \widetilde{e}_{\mathrm{c}}\right)^{2}-2 C\left({ }^{t} \widetilde{e} / \widetilde{e}_{\mathrm{c}}\right)^{3}\right]}{\left[1+A\left(\widetilde{e} / \widetilde{e}_{\mathrm{c}}\right)+B\left({ }^{\tau} \widetilde{e} / \widetilde{e}_{\mathrm{c}}\right)^{2}+C\left(\widetilde{e} / \widetilde{e}_{\mathrm{c}}\right)^{3}\right]^{2}}
$$

where

$$
\begin{aligned}
& A=\frac{\left[\widetilde{E}_{0} / \widetilde{E}_{\mathrm{u}}+\left(p^{3}-2 p^{2}\right) \tilde{E}_{0} / \widetilde{E}_{\mathrm{s}}-\left(2 p^{3}-3 p^{2}+1\right)\right]}{\left[\left(p^{2}-2 p+1\right) p\right]}, \quad B=\left[\left(2 \widetilde{E}_{0} / \widetilde{E}_{\mathrm{s}}-3\right)-2 A\right], \\
& C=\left[\left(2-\widetilde{E}_{0} / \widetilde{E}_{\mathrm{s}}\right)+A\right],
\end{aligned}
$$

and the strength parameters $\widetilde{E}_{0}, \widetilde{\sigma}_{\mathrm{c}}, \widetilde{e}_{\mathrm{c}}, \widetilde{E}_{\mathrm{s}}=\widetilde{\sigma}_{\mathrm{c}} / \widetilde{e}_{\mathrm{c}}, \widetilde{\sigma}_{\mathrm{u}}, \widetilde{e}_{\mathrm{u}}, p=\widetilde{e}_{\mathrm{u}} / \widetilde{e}_{\mathrm{c}}$ and $\widetilde{E}_{\mathrm{u}}=\widetilde{\sigma}_{\mathrm{u}} / \widetilde{e}_{\mathrm{u}}$ are obtained from uniaxial tests.

The stress-strain relation in eq. (10) assumes monotonic loading conditions. For unloading conditions and load. ing back to the stress state from which unloading occurred, the initial Young's modulus $\widetilde{E}_{0}$ is used.

\subsubsection{Multiaxial conditions}

The behavior of concrete and rock materials under multiaxial stress conditions is very complex and has not been assessed experimentally in a complete manner. Various material models with considerable simplifying assumptions have been proposed to characterize the behavior of concrete and rock materials using plasticity relations, hypoelastic descriptions and the endochronic theory of inelasticity [9-16]. However, considering the variability of concrete materials that need be described in practice, and recognizing that the model should also be useful to represent the behavior of some rock materials, the objective in this work was to develop an effective but simple 
model that provides sufficient flexibility to the analyst to fit basic material behaviors.

The stress-strain relations are evaluated differently depending on whether the material is loading or unloading. The Poisson ratio is assumed to be constant under all stress conditions.

To characterize loading and unloading conditions we define a loading function

$$
{ }^{t} f={ }^{t} \bar{s}+3 \alpha^{t} \sigma_{m},
$$

where $\alpha$ is a constant (usually negative), ${ }^{t} \sigma_{m}=\frac{1}{3}{ }^{t} \sigma_{i i},{ }^{t} \bar{s}=\left(\frac{1}{2}{ }^{t} s_{i j}{ }^{t} s_{i j}\right)^{1 / 2},{ }^{t} s_{i j}={ }^{t} \sigma_{i j}-\delta_{i j}{ }^{t} \sigma_{m}$ and $\delta_{i j}$ is the Kronecker delta. The material is loading if

$$
{ }^{t} f \geqslant f_{\max }
$$

and unloading if

$$
{ }^{t} f<f_{\max },
$$

where $f_{\max }$ is the maximum value of the loading function that has been reached during the complete solution. In unloading, the material is assumed to be isotropic and the initial Young's modulus, $\widetilde{E}_{0}$, is used to form the incremental stress - strain matrix, both for stiffness and stress calculations.

To obtain the stress--strain relations in loading conditions, the principal stresses are calculated and for each principal stress direction a uniaxial tangent Young's modulus, ${ }^{t} \widetilde{E}_{\mathrm{pi}}$, corresponding to the strain in the principal stress direction, ${ }^{t} e_{p i}$, is evaluated using eqs. (9) and (11). When using eq. (11), the current strain ${ }^{t} e_{p i}$ is employed and to account for multiaxial stress conditions the material variables $\widetilde{\sigma}_{\mathrm{c}}, \widetilde{\sigma}_{\mathrm{u}}, \widetilde{e}_{\mathrm{c}}$ and $\tilde{e}_{\mathrm{u}}$ are replaced by the variables $\tilde{\sigma}_{\mathrm{c}}^{\prime}, \widetilde{\sigma}_{\mathrm{u}}^{\prime}, \tilde{e}_{\mathrm{c}}^{\prime}$ and $\tilde{e}_{\mathrm{u}}^{\prime}$ defined in eq. (20). Let ${ }^{t} \sigma_{p 1},{ }^{t} \sigma_{p 2}$ and ${ }^{t} \sigma_{p 3}$ be the principal stresses at time $t$, with ${ }^{t} \sigma_{p 3} \leqslant{ }^{t} \sigma_{p 2} \leqslant$ ${ }^{t} \sigma_{p 1}$ and ${ }^{t} \widetilde{E}_{p 1},{ }^{t} \widetilde{E}_{p 2}$ and ${ }^{t} \widetilde{E}_{p 3}$ the corresponding uniaxial Young's moduli. The material is considered as isotropic with an equivalent multiaxial Young's modulus when subjected to tension or low compression, where such a state is defined by ${ }^{t} \sigma_{p 3} \geqslant \kappa \tilde{\sigma}_{c}^{\prime}$. The variable $\kappa$ is typically 0.4 . For the material the equivalent multiaxial Young's modulus, ${ }^{t} E$, is then obtained using the following weighting scheme,

$$
{ }^{t} E=\frac{\left|{ }^{t} \sigma_{p 1}\right|{ }^{t} \widetilde{E}_{p 1}+\left|{ }^{t} \sigma_{p 2}\right|{ }^{t} \widetilde{E}_{p 2}+\left|{ }^{t} \sigma_{p 3}\right|{ }^{t} \widetilde{E}_{p 3}}{\left|{ }^{t} \sigma_{p 1}\right|+\left|{ }^{t} \sigma_{p 2}\right|+\left|{ }^{t} \sigma_{p 3}\right|}
$$

and the Poisson ratio is assumed to be constant as noted earlier.

If the material is under high compression, i.e., ${ }^{t} \sigma_{p 3}<\kappa \widetilde{\sigma}_{c}^{\prime}$, an orthotropic stress--strain matrix with the directions of orthotropy defined by the principal stress directions is employed. The stress-strain matrix corresponding to these directions is, considering three-dimensional stress conditions,

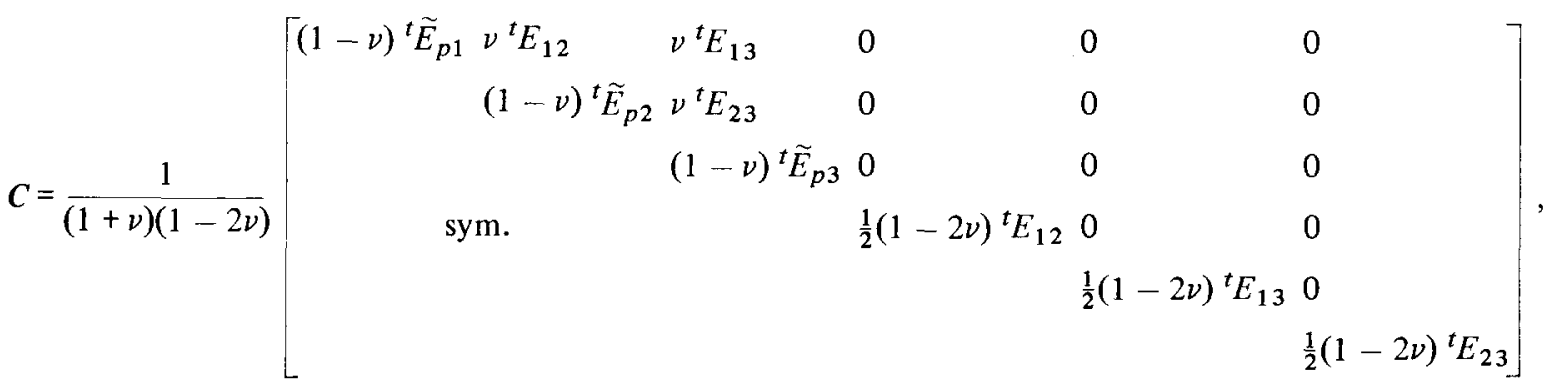

where $\nu$ is the constant Poisson ratio, and the shear modulus in a coordinate plane is calculated from the weighted Young's modulus corresponding to that plane,

$$
{ }^{t} G_{i j}=\frac{{ }^{t} E_{i j}}{2(1+\nu)}=\frac{1}{2(1+\nu)} \frac{\left|{ }^{t} \sigma_{p i}\right|{ }^{t} \widetilde{E}_{p i}+\left|{ }^{t} \sigma_{p j}\right|{ }^{t} \widetilde{E}_{p j}}{\left|{ }^{t} \sigma_{p i}\right|+\left|{ }^{t} \sigma_{p j}\right|} .
$$


The above stress-strain relations for material loading conditions are only employed in the calculation of the stiffness matrix at time $t$. Considering the evaluation of the stress increment from time $t$ to time $t+\Delta t$, the integration in eq. (7) is approximated in the following manner,

$$
\boldsymbol{\sigma}=\hat{C} e \text {. }
$$

If the material was under tension or low compression at time $t$, i.e., ${ }^{t} \sigma_{p 3} \geqslant \kappa \widetilde{\sigma}_{c}^{\prime}$, the stress-strain matrix $\hat{C}$ in eq. (17) corresponds to an isotropic material with Young's modulus ${ }^{\tau} E$ and constant Poisson ratio $\nu$,

$$
{ }^{\tau} E=\frac{\left|{ }^{t} \sigma_{p 1}\right|{ }^{\tau} \tilde{E}_{p 1}+\left|{ }^{t} \sigma_{p 2}\right|{ }^{\tau} \tilde{E}_{p 2}+\left|{ }^{t} \sigma_{p 3}\right|{ }^{\tau} \widetilde{E}_{p 3}}{\left|{ }^{t} \sigma_{p 1}\right|+\left|{ }^{t} \sigma_{p 2}\right|+\left|{ }^{t} \sigma_{p 3}\right|} .
$$

In eq. (18), the uniaxial Young's moduli ${ }^{\tau} \widetilde{E}_{p i}$ corresponding to the current strain increment $e$ are evaluated using the uniaxial stress-strain relationship in fig. 1 ,

$$
{ }^{\tau} \widetilde{E}_{p i}=\left\{\left.\tilde{\sigma}\right|_{\text {at }}{ }^{t} e_{p i}+e_{p i}-\left.\tilde{\sigma}\right|_{\text {at }}{ }^{t} e_{p i}\right\} / e_{p i},
$$

where the ${ }^{t} e_{p i}$ and $e_{p i}$ are the strain components and incremental strain components at time $t$ measured in the directions of the principal stresses ${ }^{t} \sigma_{p i}$.

If the material was under high compression at time $t$, the stress-strain matrix employed in eq. (17) is the one defined in eq. (15) but using the Young's moduli ${ }^{\tau} \widetilde{E}_{p i}$ given in eq. (19). Also, in this case the stress and strain vectors in eq. (17) must correspond to the axes of orthotropy used in eq. (15).

\subsection{Material failure envelopes}

To model the failure of the material in tension and compression in two and three-dimensional analysis and to account for multiaxial conditions in the uniaxial stress-strain behavior, failure envelopes are employed. Based on the current knowledge of concrete material behavior, the tensile and compression failure envelopes shown in figs. 2 and 3 have been implemented. The tensile failure envelope given in fig. 2 is commonly employed. It is noted that

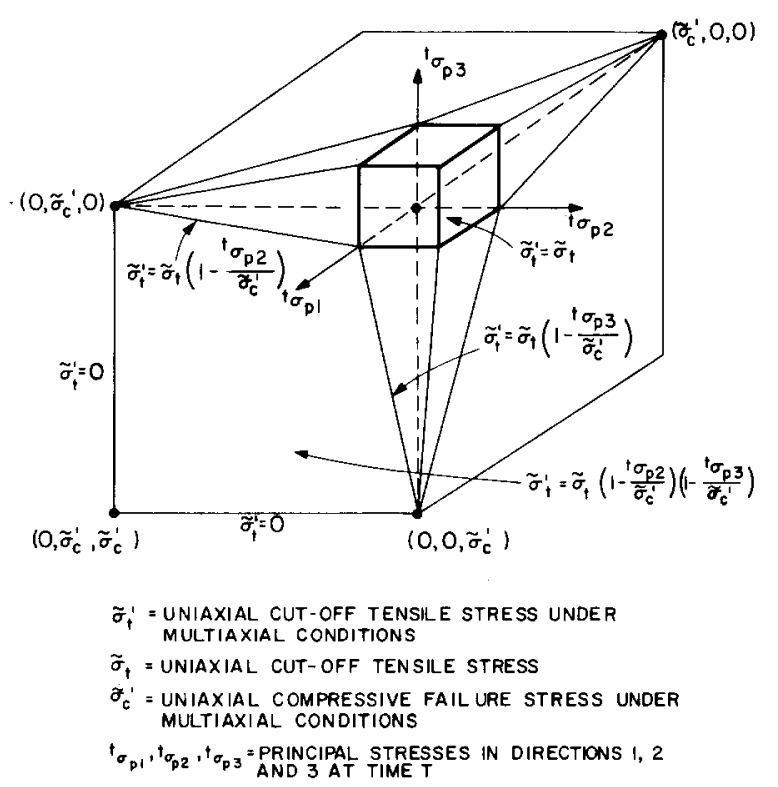

Fig. 2. Triaxial tensile failure envelope of model. 


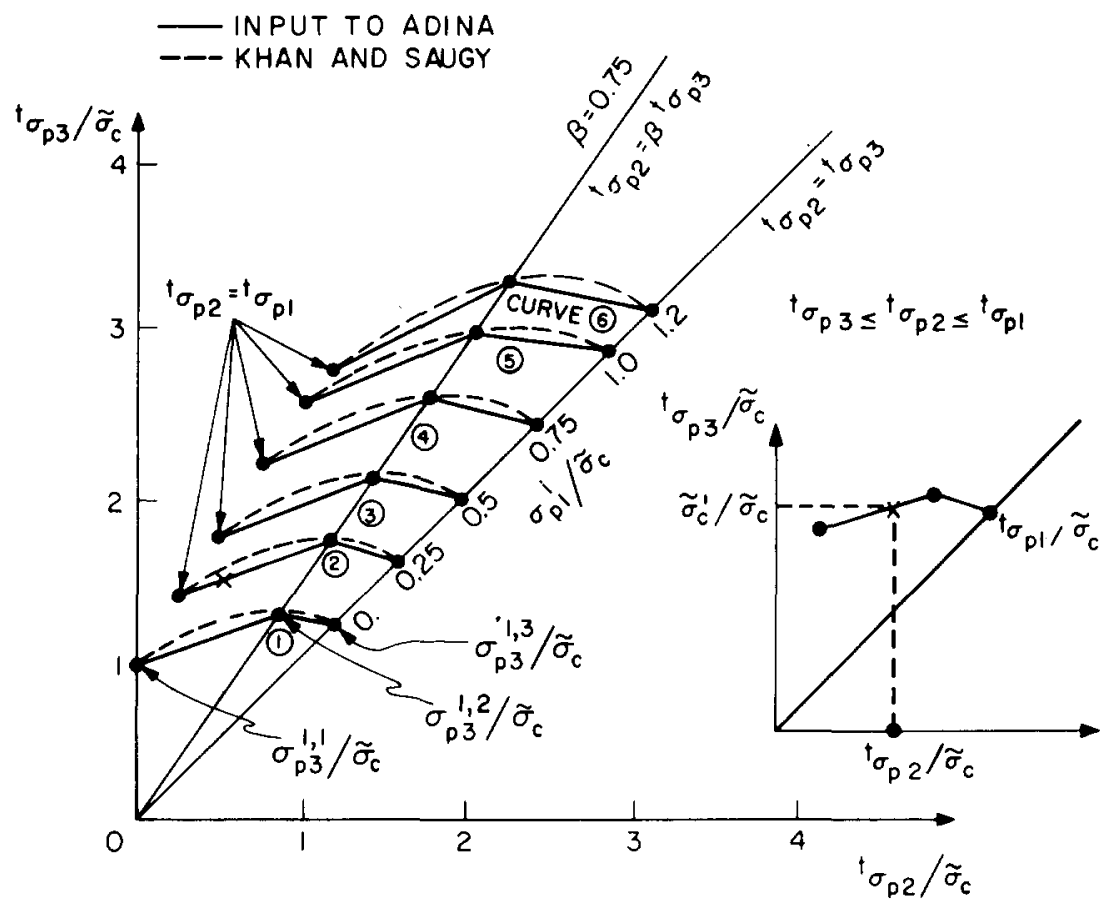

Fig. 3. Triaxial compressive failure envelope of model.

considering one principal stress direction the tensile strength of the material in this direction does not change with the introduction of tensile stresses in the other principal stress directions, but compressive stresses change this tensile strength.

Considering the compression failure envelope, it should be noted that the failure envelope shown in fig. 3 can be used to represent a large number of different envelopes like the biaxial envelope of Liu et al. [7] and the triaxial failure surface of Khan and Saugy [8]. The shape of the compressive failure surface used is largely based on the experimental results reported by Kupfer et al. [6] and Launay and Gachon [9], but the flexibility provided in the envelopes used here makes it possible to model various concrete and rock materials. The envelope can be employed to model a Mohr-Coulomb or Drucker-Prager failure surface.

The compression failure envelope is input using 24 discrete stress values. Firstly, the values $\sigma_{p 1}^{i} / \widetilde{\sigma}_{\mathrm{c}}$ are input. These values define at what stress magnitudes ${ }^{t} \sigma_{p 1}$ the discrete two-dimensional failure envelopes for additional stresses ${ }^{t} \sigma_{p 2}$ and ${ }^{t} \sigma_{p 3}$ are input. These failure envelopes are defined by the failure stress values $\sigma_{p 3}^{i, j} / \widetilde{\sigma}_{\mathrm{c}}(i=1, \ldots, 6$; $j=1,2,3)$ that correspond to the stress magnitudes ${ }^{t} \sigma_{p 2}={ }^{t} \sigma_{p 1},{ }^{t} \sigma_{p 2}=\beta^{t} \sigma_{p 3}(\beta$ is a constant $)$ and ${ }^{t} \sigma_{p 2}={ }^{t} \sigma_{p 3}$.

The failure envelopes are employed to establish the uniaxial stress-strain iaw accounting for multiaxial stress conditions, and to identify whether tensile or crushing failure of the material has occurred. Having established the current principal stresses, to establish the uniaxial stress-strain law it is assumed that ${ }^{t} \sigma_{p 1}$ and ${ }^{t} \sigma_{p 2}$ are held constant and the minimum stress that would have to be reached in the third principal stress direction to cause crushing of the material is calculated using the failure envelopes, see fig. 3. Let this stress be $\sigma_{c}^{\prime}$, and $\gamma_{1}=\tilde{\sigma}_{c}^{\prime} / \tilde{\sigma}_{c}$, then we also use

$$
\tilde{\sigma}_{\mathrm{u}}^{\prime}=\gamma_{1} \tilde{\sigma}_{\mathrm{u}} ; \quad \tilde{e}_{\mathrm{c}}^{\prime}=\gamma_{1} \gamma \tilde{e}_{\mathrm{c}} ; \quad \tilde{e}_{\mathrm{u}}^{\prime}=\gamma_{1} \gamma \widetilde{e}_{\mathrm{u}}
$$

where $\gamma$ is a constant. The constants $\tilde{\sigma}_{\mathfrak{c}}^{\prime}, \widetilde{\sigma}_{\mathbf{u}}^{\prime}, \tilde{e}_{\mathfrak{c}}^{\prime}, \tilde{e}_{\mathrm{u}}^{\prime}$ are employed instead of the unprimed variables in order to establish using eq. (11), the uniaxial stress-strain law under multiaxial conditions (see fig. 4).

To identify whether the material has failed, the principal stresses are used to locate the current stress state in 


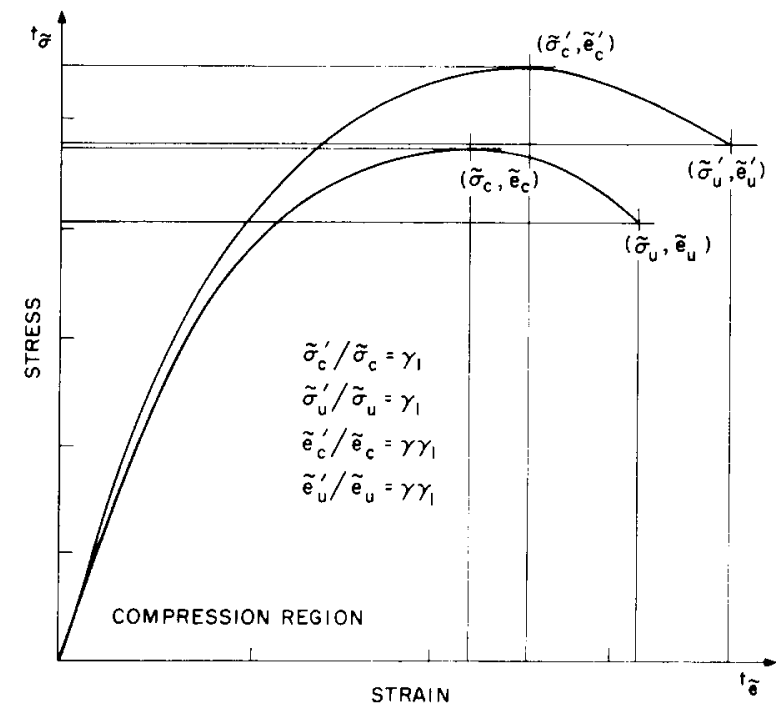

Fig. 4. Increase of strength parameters for model under multiaxial conditions.

the failure envelopes. In the following we consider how one single plane of tensile failure develops and how the material fails in compression crushing.

Tensile failure occurs if the tensile stress in a principal stress direction exceeds the tensile failure stress. In this case it is assumed that a plane of failure develops perpendicular to the principal stress direction. The effect of this material failure is that the normal and shear stiffnesses across the plane of failure are reduced, and the corresponding normal stress is released (see fig. 5). Assume that the material is subjected to low compression conditions, then before tensile failure the stress-strain relation is, considering three-dimensional analysis,

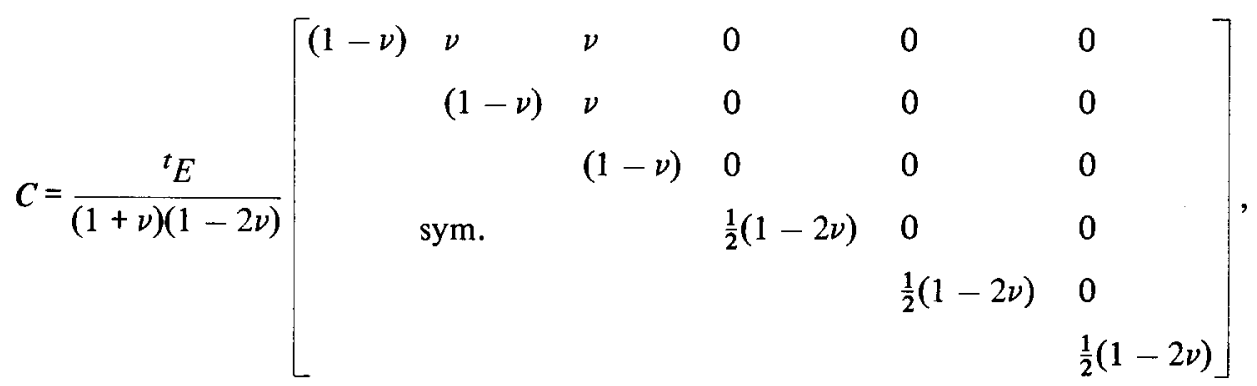

where ${ }^{t} E$ was evaluated in eq. (14). Assuming that ${ }^{t} \sigma_{p 1}$ is larger than the tensile failure stress, the new material stress-strain relation is

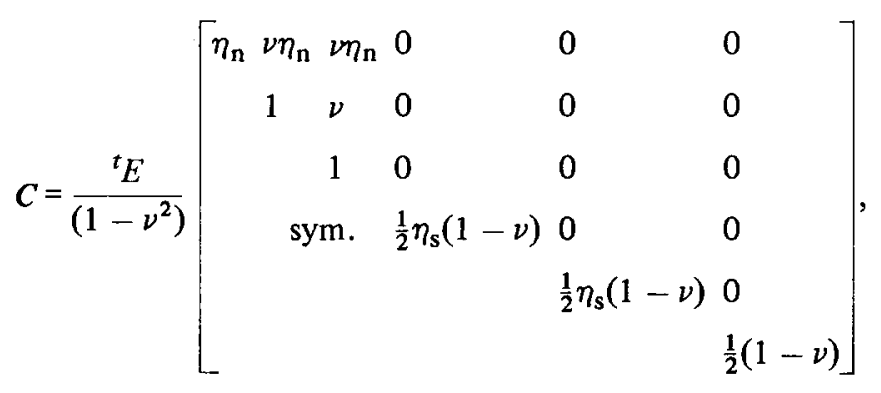



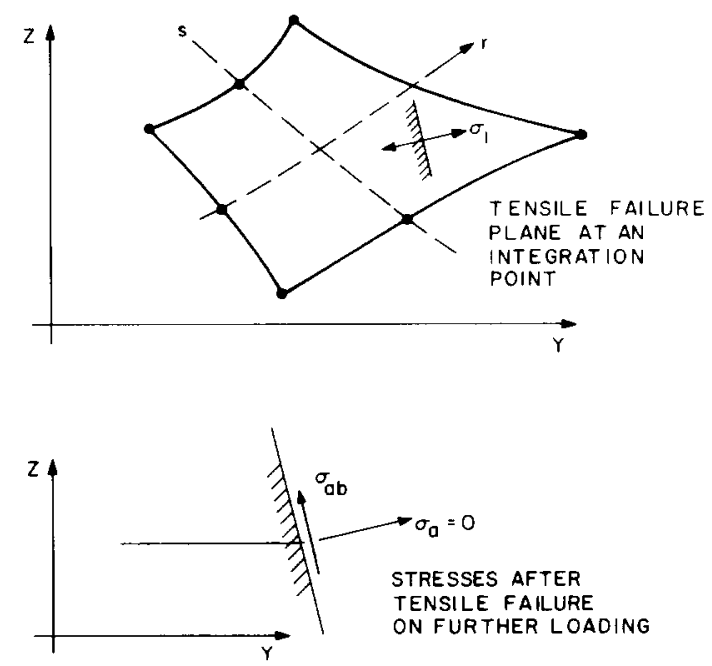

Fig. 5. Illustration of tensile failure at an integration point.

where, typically, $\eta_{\mathrm{n}}=0.001$ and $\eta_{\mathrm{s}}=0.5$, and it should be noted that plane stress conditions are assumed to exist at the plane of tensile failure. The factor $\eta_{\mathbf{n}}$ is not set exactly equal to zero in order to avoid the possibility of a singular stiffness matrix. The value to be employed for $\eta_{\mathrm{s}}$ must depend on a number of physical factors, and further research is necessary to determine appropriate values $[16,22]$. In the numerical solution it is at this point best to leave $\eta_{\mathrm{n}}$ and $\eta_{\mathrm{s}}$ as variables that are input at the start of solution.

In previous publications, the "plane of tensile failure" has been referred to as a "crack" $[13,16]$, but we choose not to employ this terminology because a physical crack does not actually develop at the element integration point. Instead, the material has failed in one principal stress direction.

If the material is subjected to high compression in orthogonal principal directions, i.e. ${ }^{t} \sigma_{p 3}<\kappa \tilde{\sigma}_{c}^{\prime}$, the same solution procedure is followed to incorporate a tensile failure, but the matrix $C$ in eq. (21) is replaced by the matrix given in eq. (15).

Considering the loading function in eq. (12) to describe loading or unloading of the material, we note that after a tensile failure in loading $f_{\max }$ is set equal to the value of the loading function corresponding to the stress state in which the stress release has been taken into account.

Eqs. (21) and (22) describe the solution when tensile failure occurs. To identify compression failure, the largest principal stress ${ }^{t} \sigma_{p 1}$ is employed to establish from fig. 3 , by interpolation, the biaxial failure envelope on ${ }^{t} \sigma_{p 2}$ and ${ }^{t} \sigma_{p 3}$. The material has crushed if the stress state corresponding to ${ }^{t} \sigma_{p 2}$ and ${ }^{t} \sigma_{p 3}$ lies on or outside this biaxial failure envelope.

\subsection{Post tensile cracking and post compression crushing behavior}

Once a tensile plane of failure has formed, it is checked in each subsequent solution step whether the failure is still active. The failure is considered to be inactive provided the normal strain across the plane becomes negative and less than the strain at which the failure occurred initially and is active otherwise (see fig. 9). Therefore, a tensile failure plane may repeatedly be active and inactive.

If a tensile failure plane has developed, which may or may not be active, the material stress-strain relations are always established as described above but corresponding to the principal stress directions in the failure plane and the direction perpendicular to this plane. Hence, instead of using the stresses principal stresses and corresponding directions as done for the unfailed material, the stress conditions along and normal to the material tensile 
failure plane are used to evaluate the stress-strain matrix. Also, when a failure plane is or was active, a subsequent failure plane is assumed to form perpendicular to the direction of the one that developed first, once a normal stress along the original failure plane has reached the tensile failure stress. It follows that at any integration point, the direction of the third tensile failure plane is fixed once failure has occurred in two directions.

It may also happen that after tensile failure of the material (in one or two directions) the material fails in compression crushing, which is identified, as usual, by entering the compression crushing envelope in fig. 3 with the principal stress(es) that act(s) along the tensile failure plane(s).

If the material has crushed in compression, it is assumed that the material strain-softens into all directions until the minimum principal strain, ${ }^{t} e_{p 3}$, reaches $\widetilde{e}_{\mathrm{u}}^{t}$. When ${ }^{t} e_{p 3}$ becomes equal to $\widetilde{e}_{\mathrm{u}}^{t}$, all stresses are completely released and from then on the material has no more stiffness.

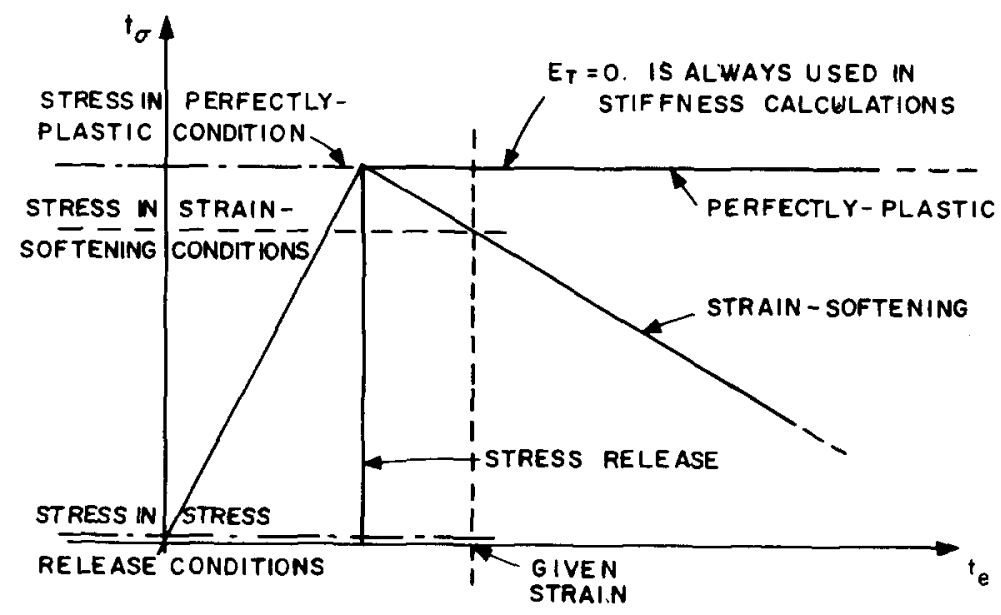

(a) ILLUSTRATION OF SOLUTION PROCEDURES FOR POST -

FAILURE ANALYSIS

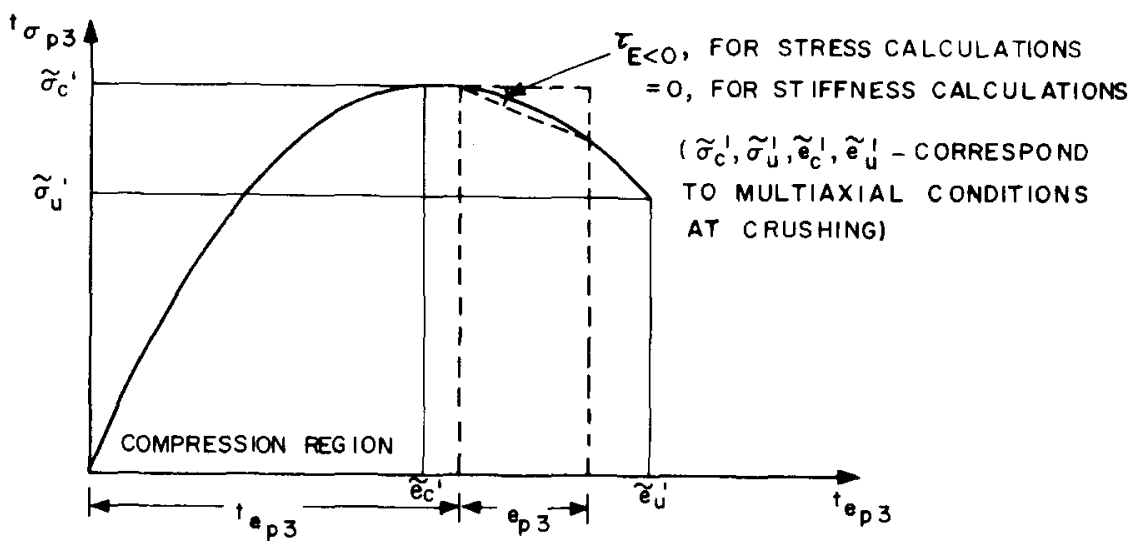

(b) SOLUTION STRATEGY ADOPTED FOR MODELING STRAIN SOFTENING CONDITIONS

Fig. 6. Strain-softening analysis procedure. 


\subsection{Strain-softening behavior}

Consider first uniaxial stress conditions. As shown in fig. 1, for a uniaxial strain smaller than $\tilde{e}_{\mathrm{c}}^{\prime}$, the material has crushed and softens with increasing compressive strain, i.e. ${ }^{t} \widetilde{E}$ is negative. The difficulty of including this material behavior lies in that the stiffness matrix can become indefinite if a negative Young's modulus is used. The solution of the finite element equations can become difficult and subject to relatively large errors when the stiffness matrix of the element assemblage is not positive definite. To circumvent this difficulty if ${ }^{t} \widetilde{E}$ in eq. (11) is negative, in this study a zero value (actually a small positive value) for ${ }^{t} \widetilde{E}$ is employed instead. However, in the calculation of the stress increments the actual negative value of ${ }^{t} \widetilde{E}$ is used. It should be noted that this solution strategy, as illustrated in fig. 6 , is a direct generalization of the common incremental procedures employed to analyze perfectly-plastic conditions and conditions of complete stress release $[10,20]$.

Under multiaxial stress conditions the compression crushing is identified using the multiaxial failure envelope, and once the material has crushed isotropic conditions are assumed. As in uniaxial conditions, in the subsequent solution steps the Young's modulus is assumed to be zero in the stiffness matrix calculations, but the stress increments are computed from the uniaxial stress-strain law with the constants $\widetilde{\sigma}_{\mathrm{c}}^{\prime}, \widetilde{e}_{\mathrm{c}}^{\prime}$ and so on (see fig. 4) corresponding to the multiaxial conditions at crushing. The Young's modulus ${ }^{\tau} E$ corresponding to the current strain increment $e_{p 3}$ is evaluated using the uniaxial stress-strain relationship in fig. 1,

$$
{ }^{\tau} E=\left\{\left.\tilde{\sigma}\right|_{\text {at }}{ }^{t} e_{p 3}+e_{p 3}-\left.\tilde{\sigma}\right|_{\text {at }}{ }^{t} e_{p 3}\right\} / e_{p 3},
$$

where the ${ }^{t} e_{p 3}$ and $e_{p 3}$ are the strain component and incremental strain component at time $t$ measured in the direction of the principal stress ${ }^{t} \sigma_{p 3}$. To obtain the stress increment, eq. (17) is used where the matrix $\hat{C}$ corresponds to isotropic material conditions with Young's modulus ${ }^{\top} E$.

If unloading of the crushed material in the strain-softening region occurs, characterized by $e_{p 3} \geqslant 0$, the stress increments are assumed to be zero.

\section{Computer implementation of material model}

The material model has been implemented in the computer program ADINA [17,21]. The following material model parameters have to be input to the program:

(a) the uniaxial stress-strain law parameters $\widetilde{E}_{0}, \widetilde{\sigma}_{\mathrm{t}}, \widetilde{\sigma}_{\mathrm{c}}, \tilde{e}_{\mathrm{c}}, \widetilde{\sigma}_{\mathrm{u}}, \tilde{e}_{\mathrm{u}}$, defined in section 3.1 ;

(b) the constant Poisson ratio, $\nu$;

(c) the triaxial compressive failure envelope defined by six values $\sigma_{p 1}^{i} / \widetilde{\sigma}_{\mathrm{c}}$, eighteen values $\sigma_{p 3}^{i, j} / \widetilde{\sigma}_{\mathrm{c}}$ and the constant $\beta$, described in section 3.2 and shown in fig. 3 ;

(d) other analysis control parameters: $\gamma=$ a constant used for scaling $\tilde{e}_{\mathrm{c}}$ and $\tilde{e}_{\mathrm{u}}$ under multiaxial conditions, $\kappa=$ a control parameter that defines when to use isotropic or orthotropic stress-strain relations, $\alpha=$ a constant multiplier for the hydrostatic component in the loading function; these three parameters are described in section 3.1; $\eta_{\mathrm{n}}, \eta_{\mathrm{s}}=$ normal and shear stiffness reduction factors, defined in section 3.2 .

Considering an incremental analysis, the complete solution for the calculation of the stresses and the stressstrain relations is summarized in table 2 .

The basic equation considered in this table is,

$$
{ }^{t+\Delta t} \sigma=C e+{ }^{t} \sigma \text {. }
$$

Assuming that all stresses and the tensile failure and crushing conditions at time $t$ are known, the table summarizes the evaluation of $C$ and ${ }^{t+\Delta t} \sigma$. It should be noted that the stresses at time $t+\Delta t$ are calculated using, firstly, the material moduli corresponding to the current strain increment and secondly, any new conditions of tensile failure and crushing that have to be taken into account.

In some analyses temperature strains, $e^{\text {th }}$, and creep strains, $e^{\mathrm{c}}$, need be included. These strains can be taken 
Computer implementation of concrete and rock material model

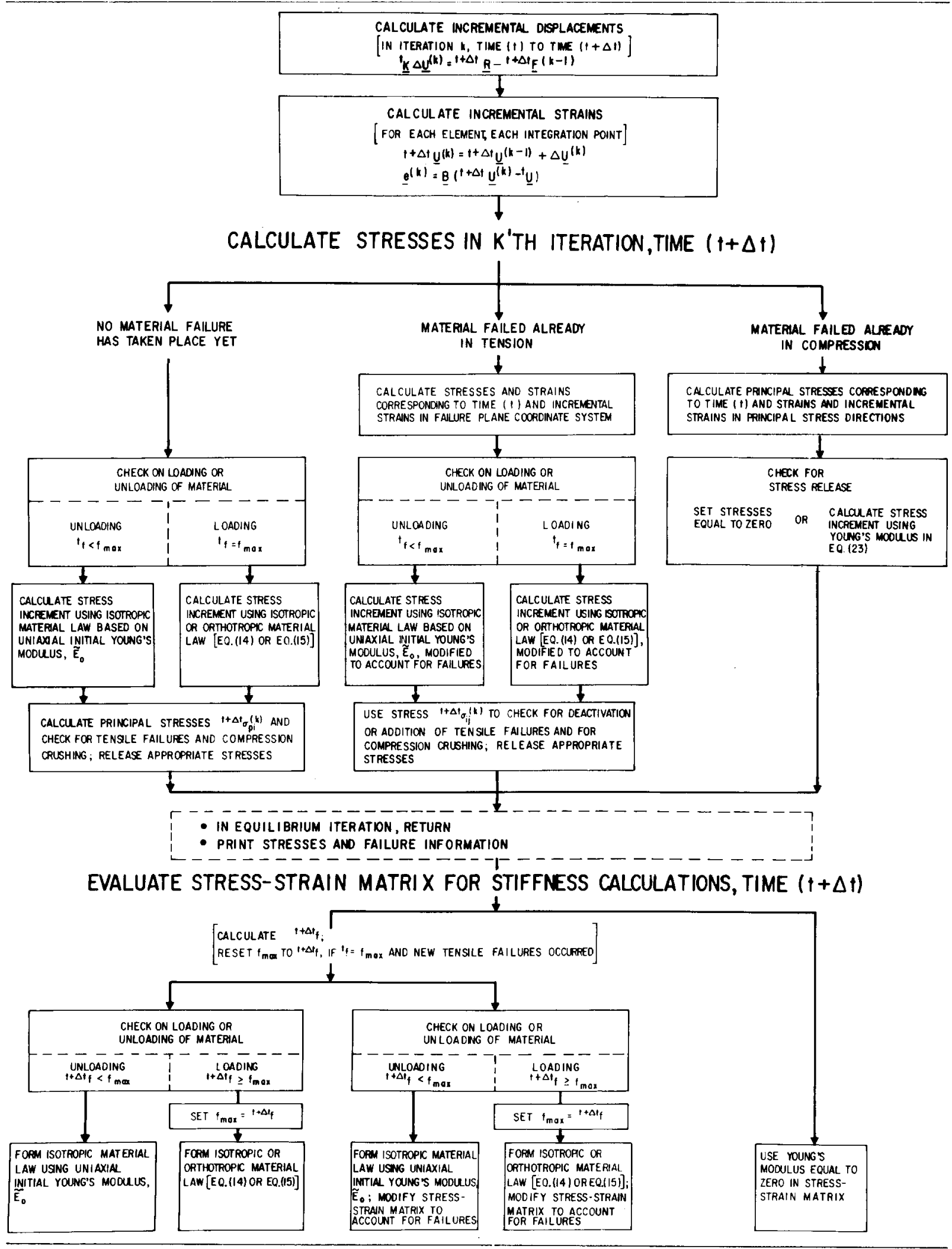


into account by replacing the total incremental strain, $e$, in eq. (3) (and in table 2) by the strain increment, $e-$ $e^{\text {th }}-e^{\mathrm{c}}$, where $\boldsymbol{e}^{\text {th }}$ and $\boldsymbol{e}^{\mathrm{c}}$ must be calculated depending on the temperature, stress and strain conditions [20].

\section{Modeling of steel reinforcement, prestressing and steel liners}

As a structural material, concrete is used with steel reinforcement, prestressing cables, and steel liners. In the computer program ADINA, depending on the structure to be analyzed, steel reinforcement can be modeled by discrete truss elements or plane stress elements. Steel liners are represented by plane stress elements. Prestressing cables are modeled using cable elements with initial forces. In order to represent the time lag between the application of different prestressing, an element birth option is employed in which an element becomes active only from its time of birth.

The elements available in ADINA for modeling concrete and the reinforcement are depicted in fig. 7.

\section{Sample solutions}

The model described in the previous sections has been implemented in the computer program ADINA and was used to analyze a number of problems. In this section, we report some of the solution results. In almost all the

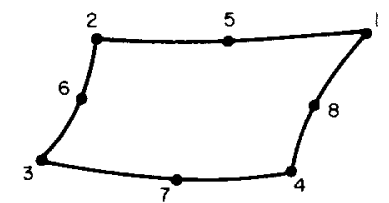

(a) TWO-DIMENSIONAL CONCRETE ANO ROCK ELEMENTS ( 3 TO 8 VARIABLE - NUMBER-NODES ELEMENTS FOR PLANE STRESS, PLANE STRAIN AND AXISYMMETRIC ANALYSES)

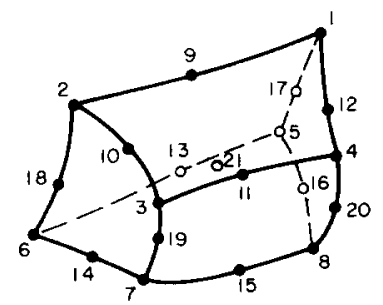

(b) THREE - DIMENSIONAL CONCRETE AND ROCK ELEMENTS ( 4 TO 21 VARIABLE-NUMBER-NODES ELEMENTS )
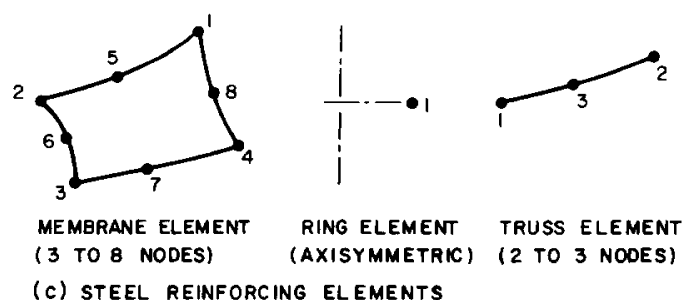

Fig. 7. Finite elements available in ADINA for analysis of concrete and rock structures. 
analyses, the material model was used to model concrete structures because some comparisons with analytical or experimental results are available.

In all the following analyses, the three-dimensional compressive failure envelope shown in fig. 3 was used.

\subsection{Demonstrative analyses of a concrete or rock sample}

Some simple loading conditions were analyzed in order to numerically identify the essential characteristics of the material model that are summarized in figs. 1-6 and to identify typical numerical solution errors. We present here the one-dimensional stress and strain response of a simple 4-node plane stress element.

Fig. 8 shows the single 4-node element which was subjected to a linearly increasing stress in the first analysis and to a strain-controlled loading in the second analysis. The comparison of the response predicted with the analytical stress-strain law (which is input to the program) shows that, as expected, in each analysis the calculated stress-strain points are on the analytical curve. In the strain-controlled analysis the strain-softening branch is also traced out.

Considering the analysis results obtained in the stress-controlled loading only the response up to the maximum stress, $\widetilde{\sigma}_{\mathrm{c}}$, could be predicted, and the strain-softening region could not be reached, because a reduction in applied stress is considered as unloading (using $\widetilde{E}_{0}$ ). In addition, fig. 8 shows that, as would be anticipated, the predicted stress is smaller than the applied stress if equilibrium iteration is not employed.

The same element was then also subjected to cyclic one-dimensional strain-controlled loading as shown in fig. 9. The response sequence shows how unloading from compression at point $A$, cracking at point $B$ with subsequent stress release to point $C$ is predicted. Next, the element was unloaded further to point $D$ and then reloaded to points $E$ and $F$ to reach the original maximum load level. Finally, the element was loaded to crushing at point $G$ and into the strain-softening region until ultimate material failure at point $\mathrm{H}$.

\subsection{Two and three-dimensional analysis of a simply-supported concrete beam}

The simply supported beam shown in fig. 10 was modeled using the following finite element idealizations: Case 1: five 12-node three-dimensio lements. Corresponding to the $X, Y, Z$ coordinate axes $2 \times 3 \times 3$

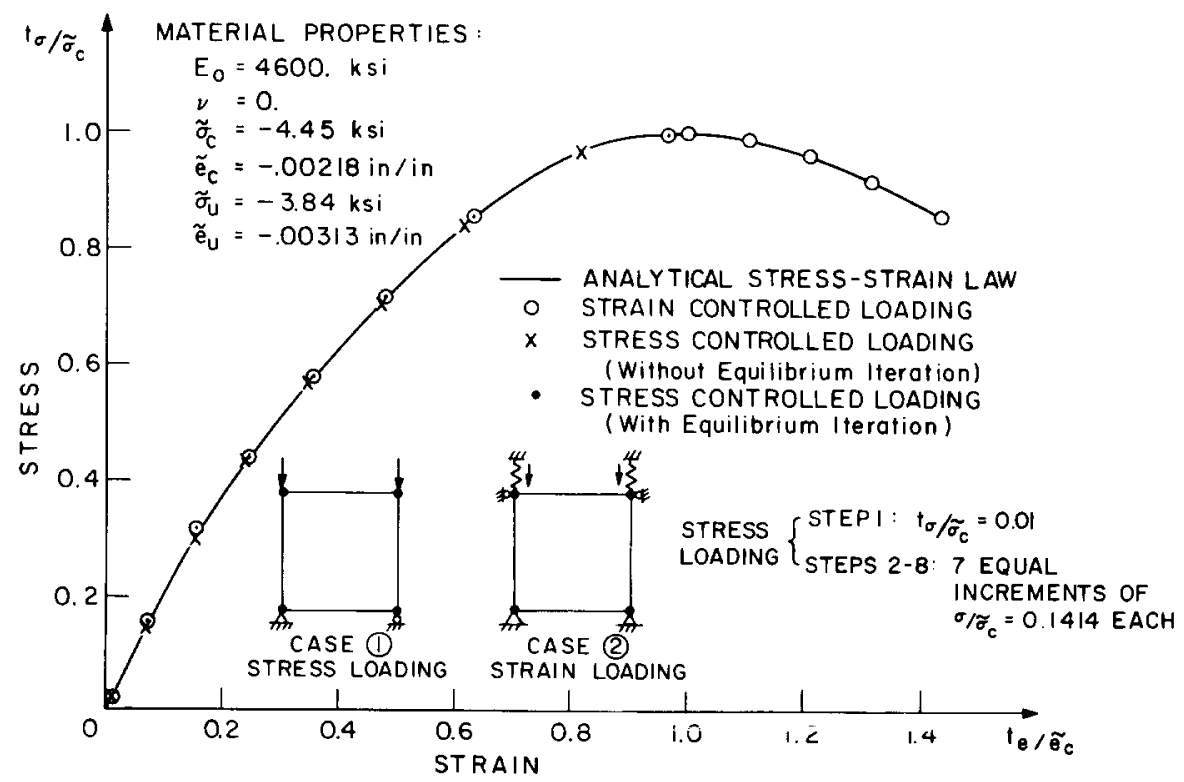

Fig. 8. Uniaxial stress-strain response of model. 


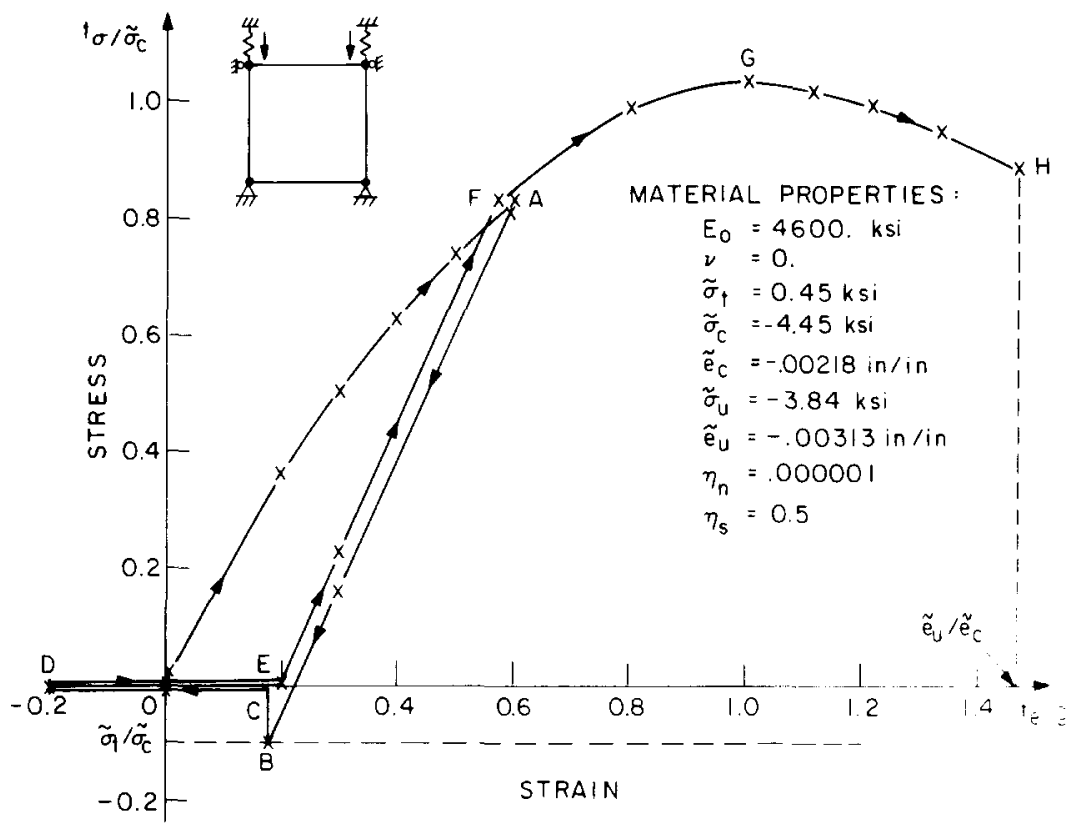

Fig. 9. Uniaxial behavior of model with unloading and reloading in compression.

Gauss numerical integration was employed. The steel reinforcement, 2 in $^{2}$, was modeled using the 6-node membrane elements.

Case 2: five 6-node two-dimensional elements with Gauss numerical integration order $3 \times 3$. The steel reinforce ment, 2 in. ${ }^{2}$, was modeled using 3 -node truss elements.

Case 3: ten 6-node two-dimensional elements with Gauss numerical integration order $2 \times 2$. The steel reinforce ment, 2 in. ${ }^{2}$, was modeled using 3 -node truss elements.

Case 4: the element idealization of case 3 was used but the steel reinforcement was 0.62 in. ${ }^{2}$.

The objective was to compare the beam response predicted in this analysis with the response calculated by Suidan and Schnobrich [13] and an analytical solution [23].

Fig. 11 shows the midspan displacement of the beam, and fig. 12 gives the maximum steel stress as a function of the applied load. It is seen that using the three different finite element idealizations of cases 1 to 3 essentially the same response is predicted. The loading procedure used is also shown in fig. 11. An average of three equilib. rium iterations were required in these analyses. The case 4 analysis results are compared in fig. 11 with the results published by Suidan and Schnobrich [13].

The zone of tensile failure at the constant moment section of the beam as a function of the applied load $P$ is shown in fig. 13, in which the response predicted by ADINA is compared with the analytical results obtained by Krahl et al. [23].

The case 3 model was also analyzed for its nonlinear dynamic response when subjected instantaneously to concentrated loads. A constant lumped mass matrix was used in the analysis. For the time integration, Newmark's method was employed. The nonlinear displacement response predicted by ADINA is shown in fig. 14 in which also the linear dynamic response and the static response are given.

\subsection{Large displacement analysis of a concrete beam}

The simply-supported concrete beam considered in the previous analysis (fig. 10) but with a span of 236 in. was analyzed including the effect of an axial load, $Q$. This axial load was applied at the centroid of the beam 

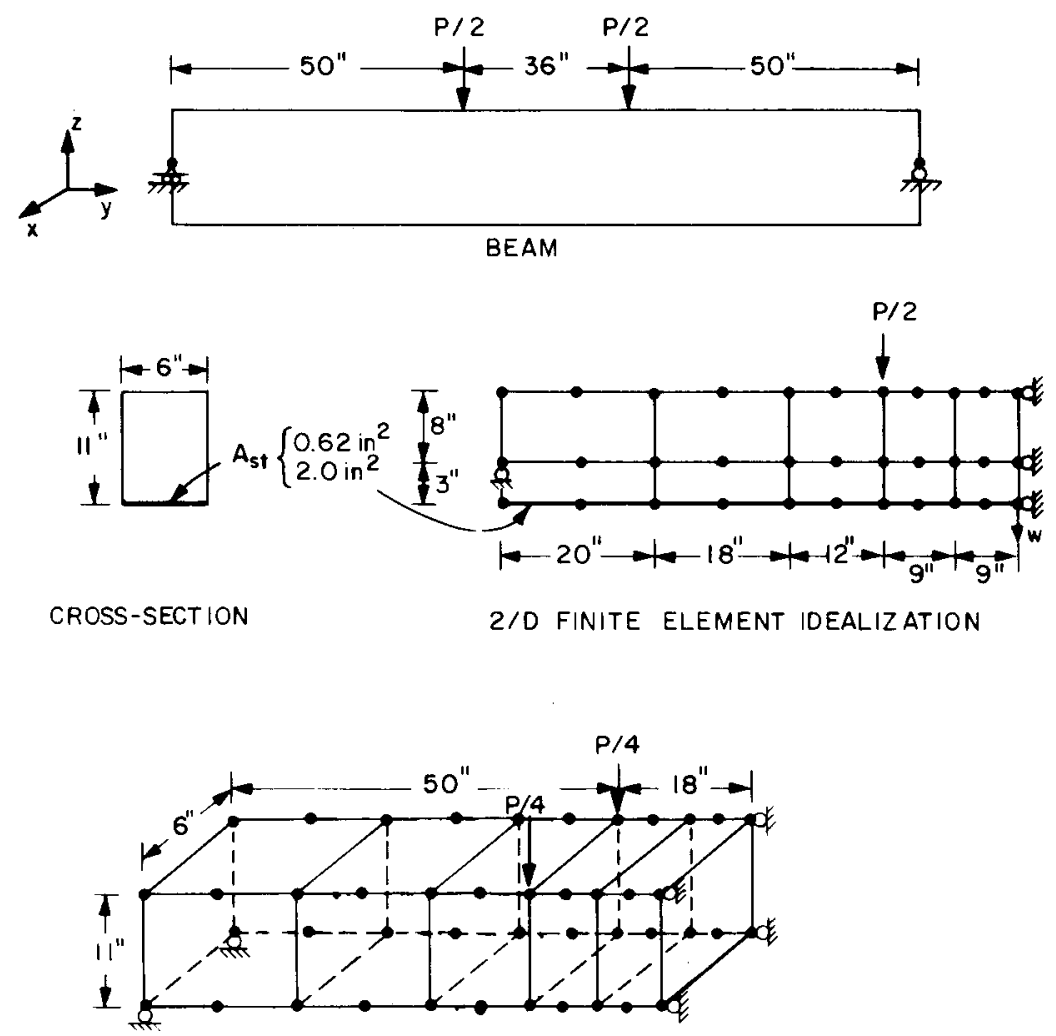

3/D FINITE ELEMENT IDEALIZATION

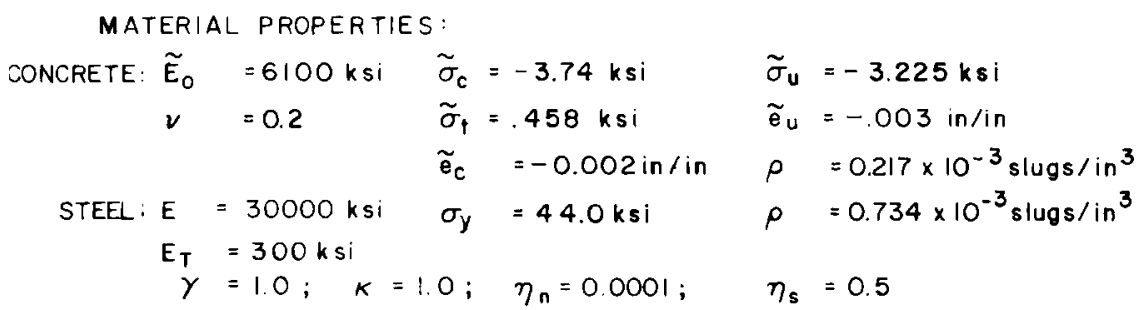

Fig. 10. Analysis of a simply-supported reinforced concrete beam.

cross-section. Fig. 15 shows the finite element model used and the predicted load displacement response of the beam with and without the axial load $Q$. In one analysis, small displacements were assumed, hence the axial load causes only a compressive normal stress in the uncracked beam; and in another analysis, large displacement effects were included. In this case, the load $Q$ also introduces a bending moment in the beam model as the structure deflects, and a smaller ultimate load is predicted. Fig. 16 shows the rebar stress at the midspan of the beam as predicted in the analyses.

Although the decrease in the ultimate load predicted in this prohlem is not drastic when including large displacement effects, the analysis does indicate that it may be important to account for large displacement effects in the analysis of some column and shell structures. 


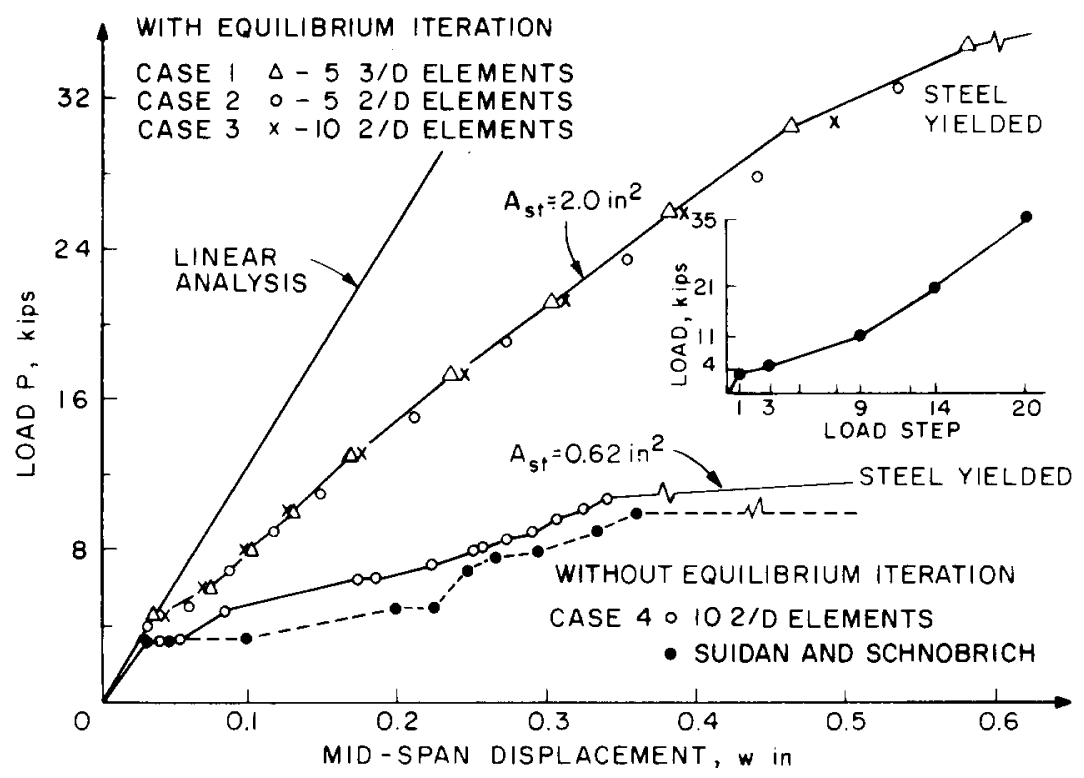

Fig. 11. Load displacement curve for the simply-supported beam.

\subsection{Analysis of a prestressed concrete reactor vessel}

The prestressed concrete reactor vessel (PCRV) for which experimentally obtained test results were reported in ref. [24] and numerical results were given in ref. [10] was analyzed. Fig. 17 shows the test vessel and the finite ele. ment mesh employed in the idealization. The different finite elements used to model the concrete and prestressing

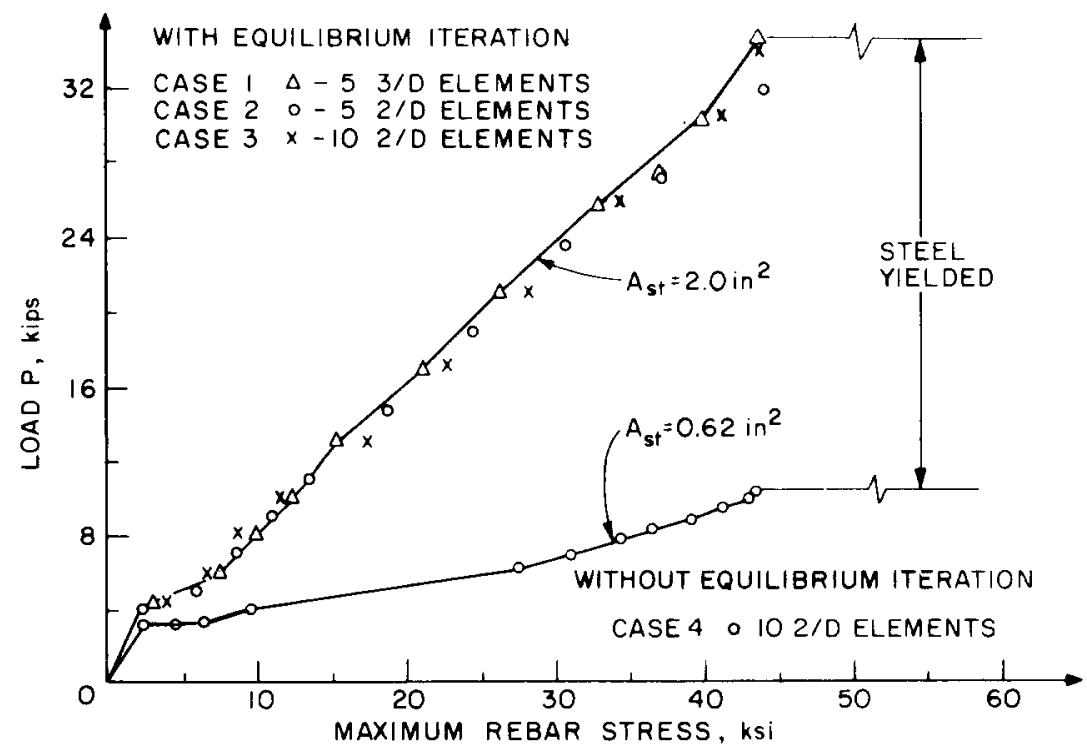

Fig. 12. Load rebar stress diagram for the simply-supported beam. 


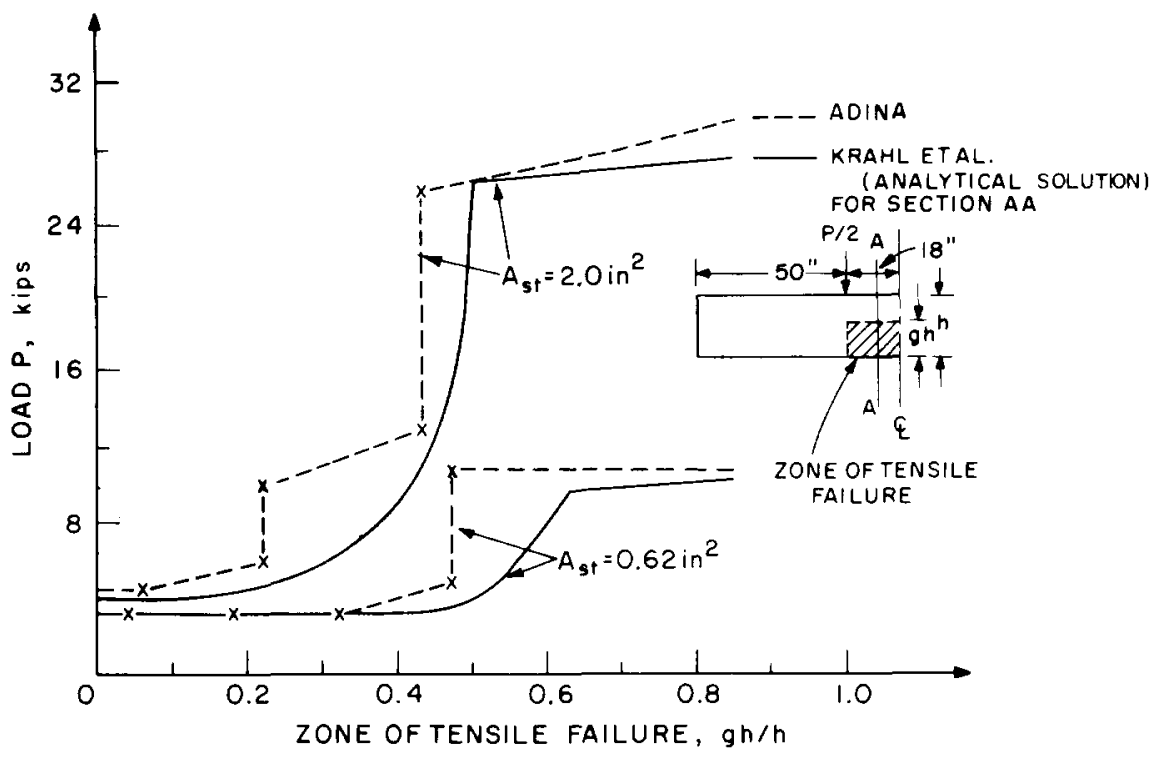

Fig. 13. Zones of tensile failure for the simply-supported beam.

cables and the material properties employed are also shown. Small displacement conditions were assumed in the analysis.

The loading procedure used in the analysis is given in fig. 18. In the first step only the circumferential prestressing of 144000 psi was applied as initial forces in the ring elements. In the next step the longitudinal prestressing

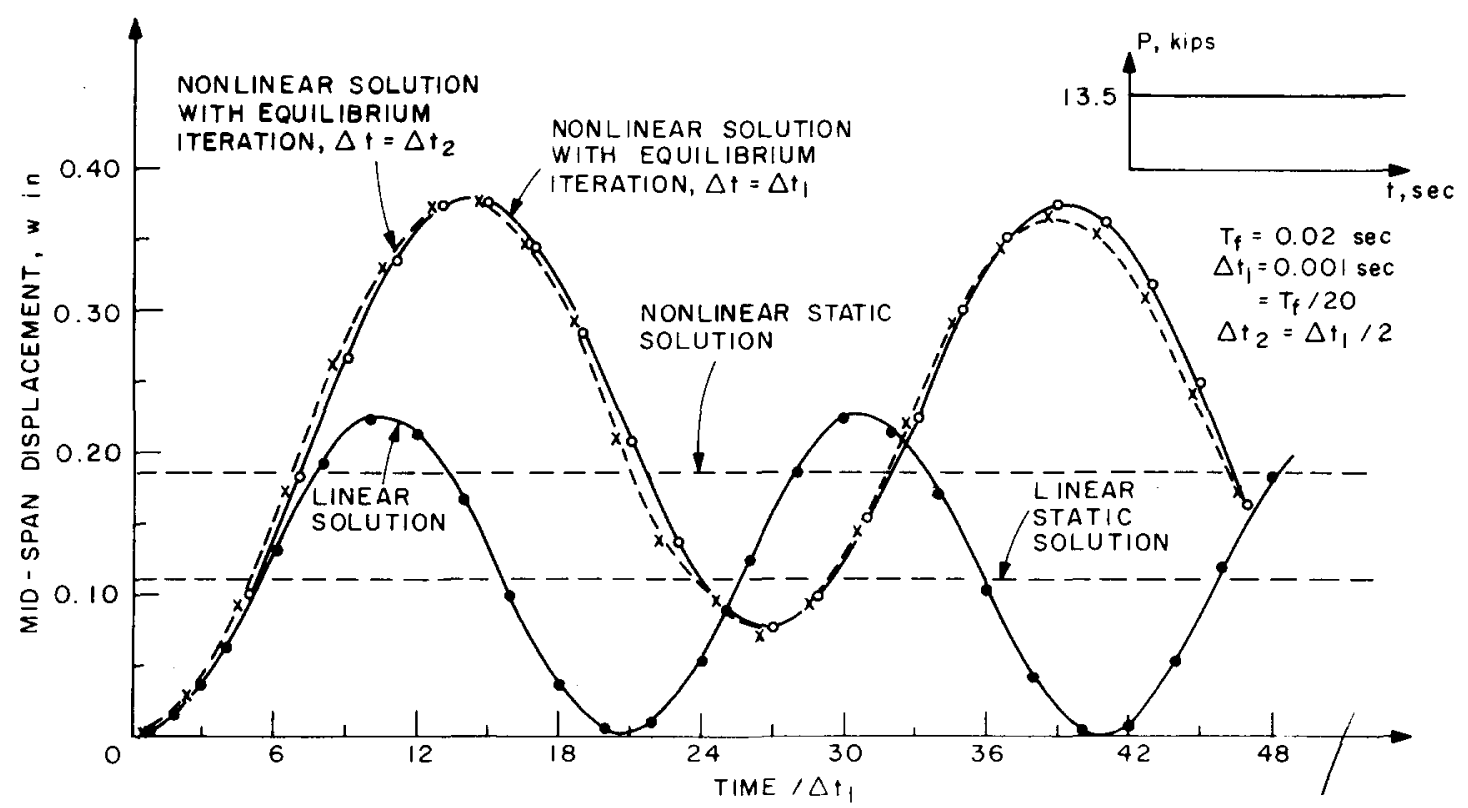

Fig. 14. Nonlinear dynamic response of the simply-supported beam, Newmark method, $\delta=0.50, \alpha=0.25$ 


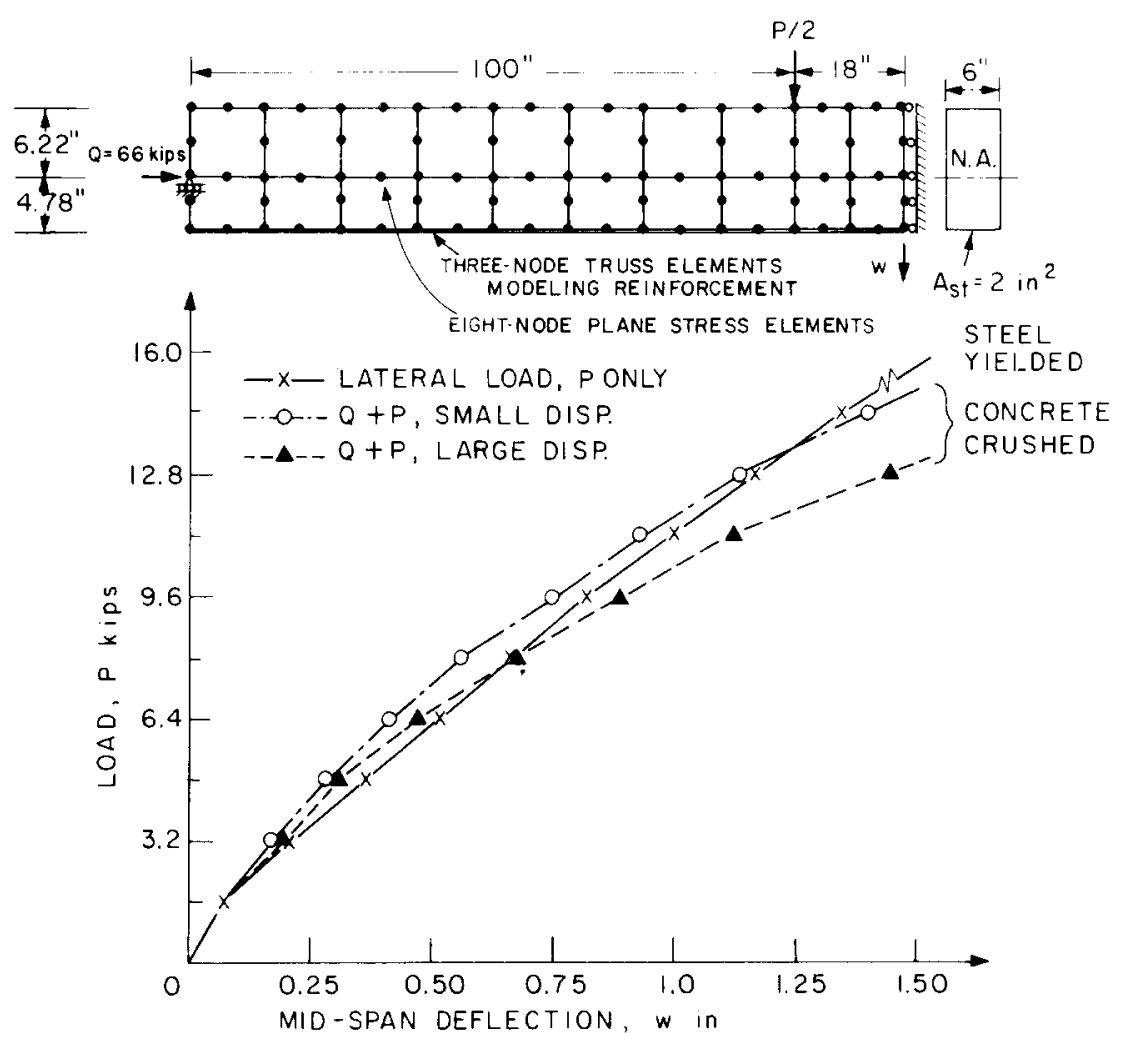

Fig. 15. Large displacement analysis of a concrete beam.

cable to introduce a prestress of 900000 psi was applied. From the third step onwards the internal pressure was applied.

Figs. 18 and 19 show the response predicted in the analysis of the vessel. Two solutions were performed with a

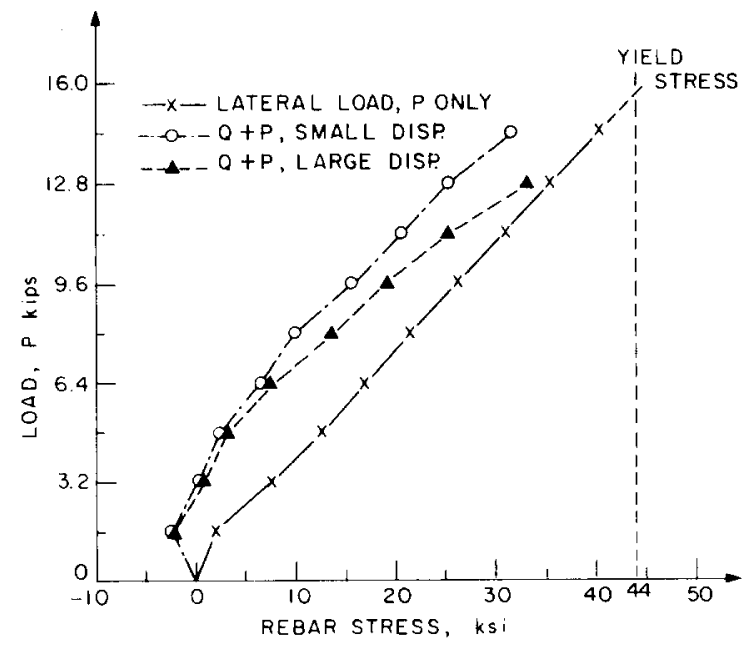

Fig. 16. Mid-span rebar stresses in the concrete beam. 


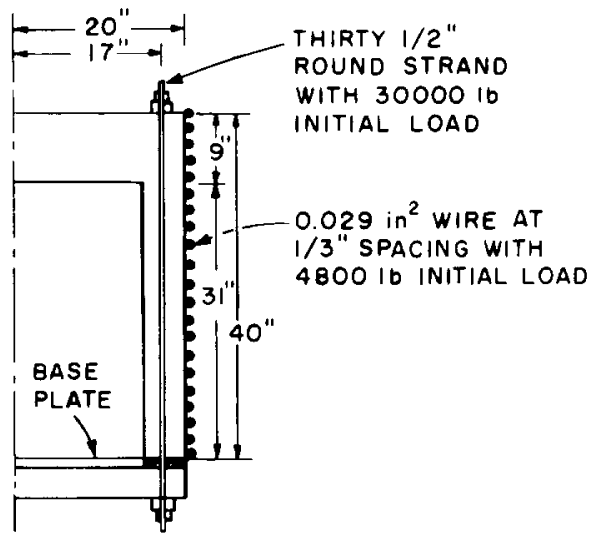

LONGITUDINAL SECTION OF THE PRESTRESSED CONCRETE REACTOR VESSEL

MATERIAL PROPERTIES:

CONCRETE:

$$
\begin{aligned}
& \tilde{E}_{0}=4300 \mathrm{ksi} \quad \tilde{\sigma}_{U}=-5.865 \mathrm{ksi} \\
& \nu=0.15 \\
& \tilde{\sigma}_{1}=0.5,0.615 \mathrm{ksi} \\
& \tilde{\sigma}_{c}=-6.9 \mathrm{ksi} \\
& \tilde{\mathbf{e}}_{\mathrm{c}}=-0.0025 \mathrm{in} / \mathrm{in} \\
& \text { STEEL: } \\
& E=28.500 \text { Rsi } \quad E_{T}=0 \\
& \sigma_{y}=225 \mathrm{ksi} \\
& \eta_{\mathrm{n}}=0.01 \\
& \eta_{\mathbf{s}}=0.5
\end{aligned}
$$
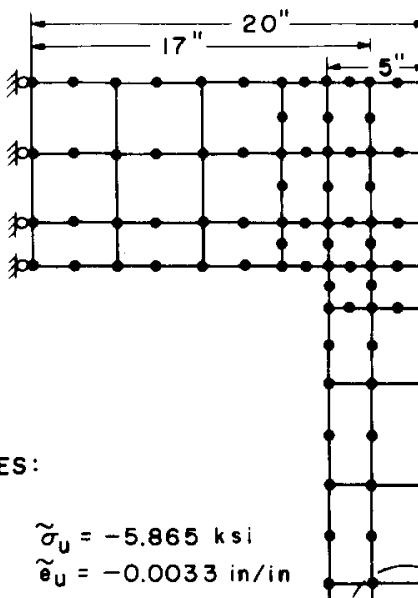

32 ISOPARAMETRIC CONCRETE ELEMENTS

21 RING ELEMENTS FOR CIRCUMFERENTIAL PRESTRESSING

I TWO-NODE TRUSS ELEMENT FOR LONGITUDINAL PRESTRESSING

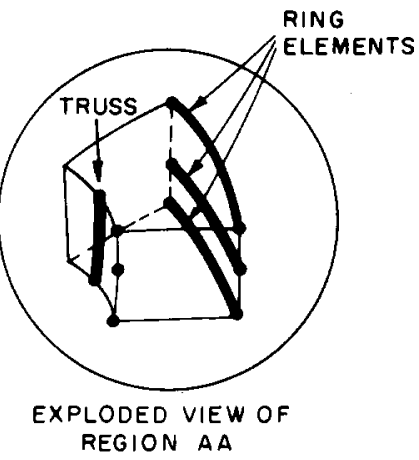

MATERIAL PROPERTES AND FINITE ELEMENT MESH

Fig. 17. Finite element model of reactor vessel.

uniaxial tensile strength of $\widetilde{\sigma}_{t}$ equal to $500 \mathrm{psi}$ and $615 \mathrm{psi}$, respectively, because the splitting test indicated a tensile strength of $\widetilde{\sigma}_{t}=500 \mathrm{psi}$ [24], whereas the ultimate tensile strain of the material indicated a tensile strength of 615 psi [10]. Fig. 18 shows that the ADINA solutions predict reasonably well the ultimate load of the structure but that the calculated displacements at the ultimate load are much too small. In the analyses, an average of two equilibrium iterations per step were performed. 


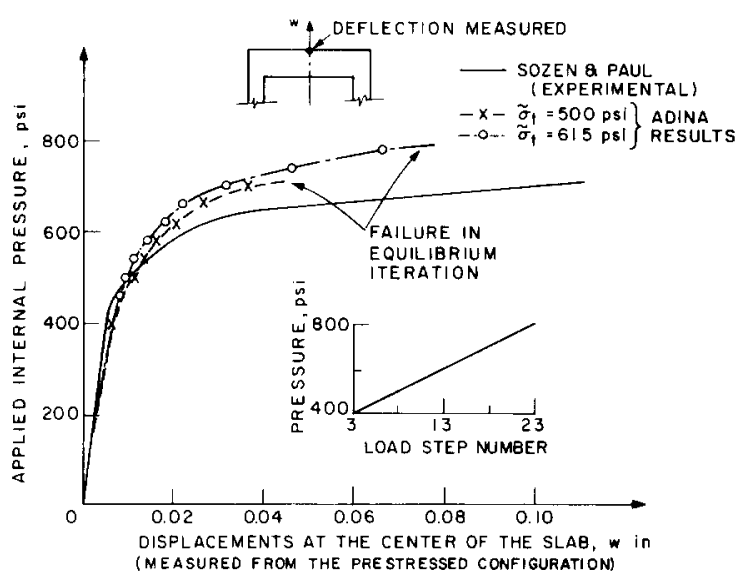

Fig. 18. Displacements of the slab at mid-span.

\subsection{Analysis of a corner supported concrete slab}

A materially nonlinear only analysis of a reinforced concrete slab was performed. The slab was supported at its four corners and subjected to a concentrated center load. Fig. 20 show's the slab and the finte element model used. It should be noted that the concrete material below the reinforcement layer was neglected in the finite element
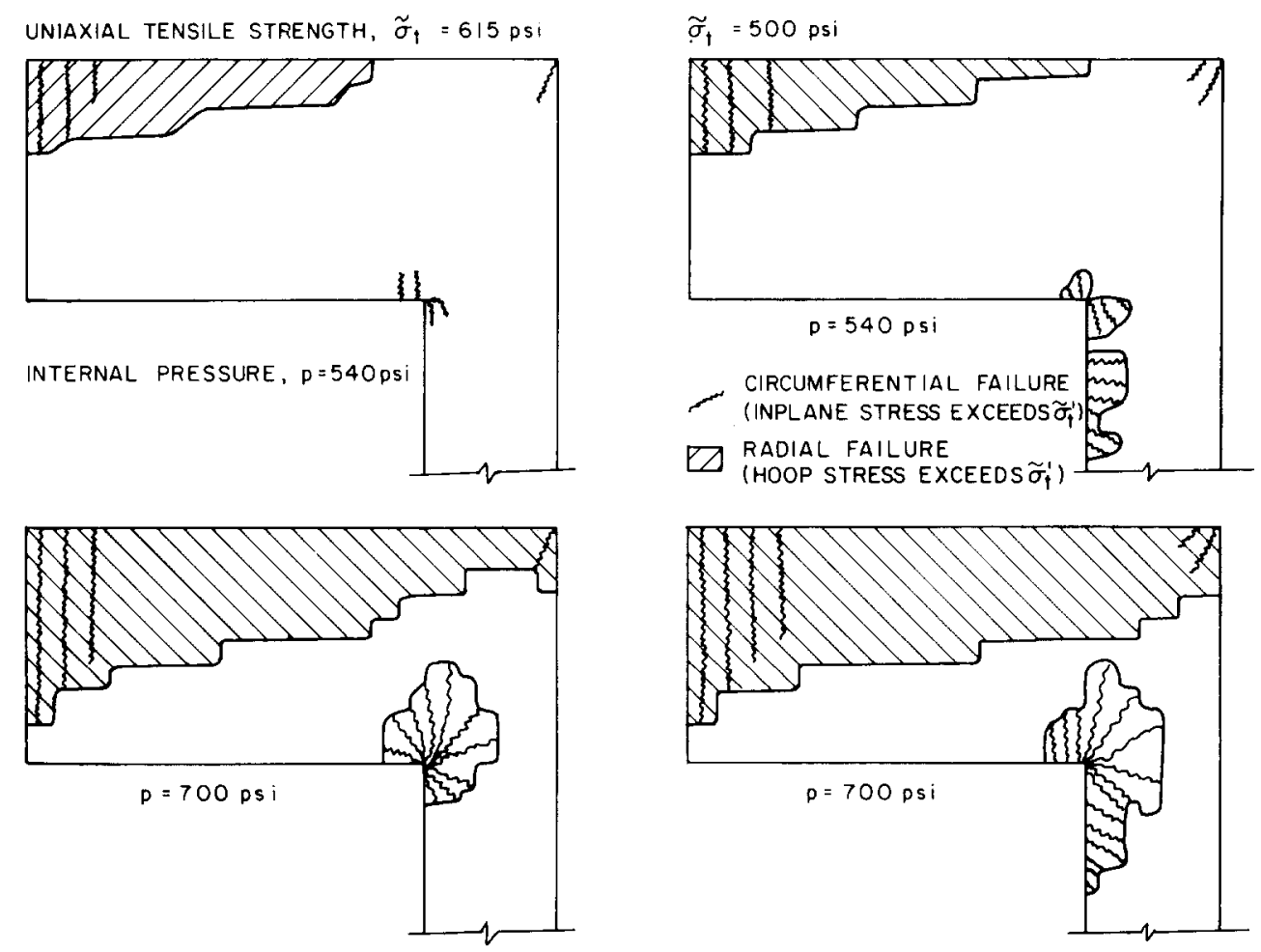

Fig. 19. Zones of tensile failure of the reactor vessel. 


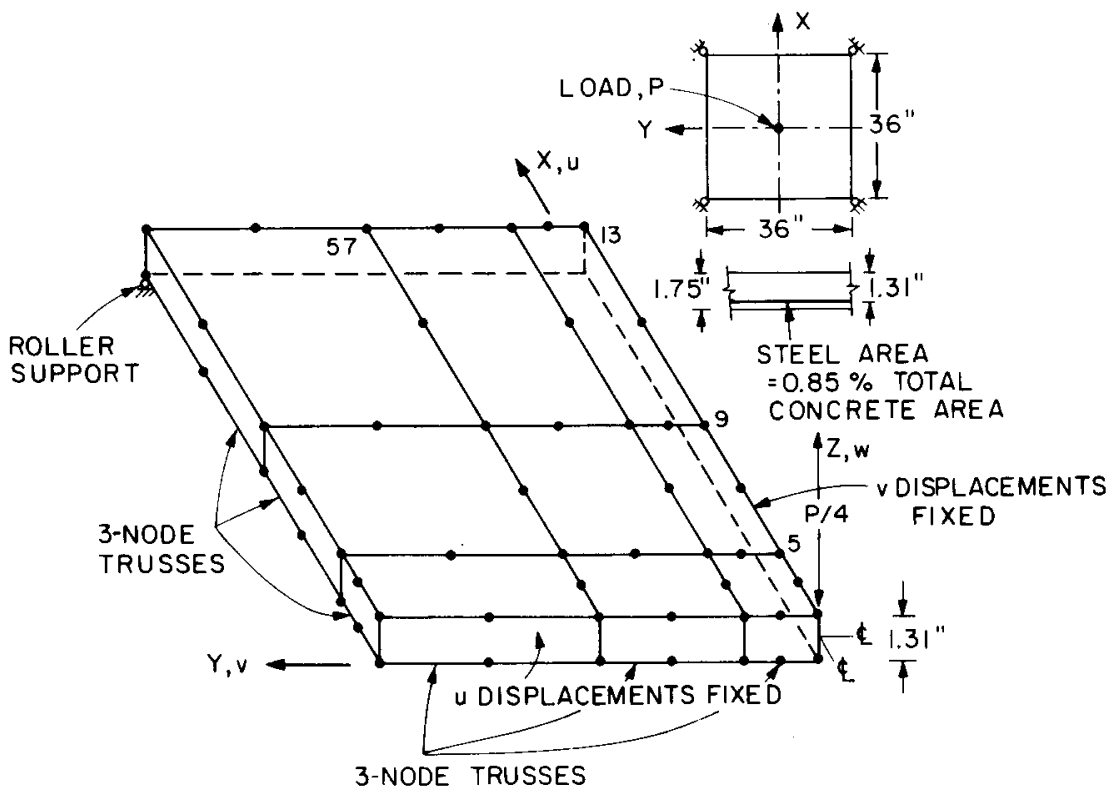

MATERIAL PROPERTIES

STEEL :

$$
\begin{aligned}
& A_{\text {st }}=0.536 \mathrm{in}^{2} \\
& E_{s t}=29000 \mathrm{ksi} \\
& \sigma_{y}=50 \mathrm{ksi} \\
& E_{T}=29 \mathrm{ksi}
\end{aligned}
$$

ANALYSIS PARAMETERS:

$\gamma=1 . ; \kappa=0.7 ; \eta_{n}=0.0001 ; \eta_{\mathrm{s}}=0.5$

9 SIXTEEN-NODE ISOPARAMETRIC ELEMENTS WITH $3 \times 3 \times 3$ INTEGRATION

24 THREE - NODE TRUSS ELEMENTS

Fig. 20. Analysis of a corner-supported concrete slab.

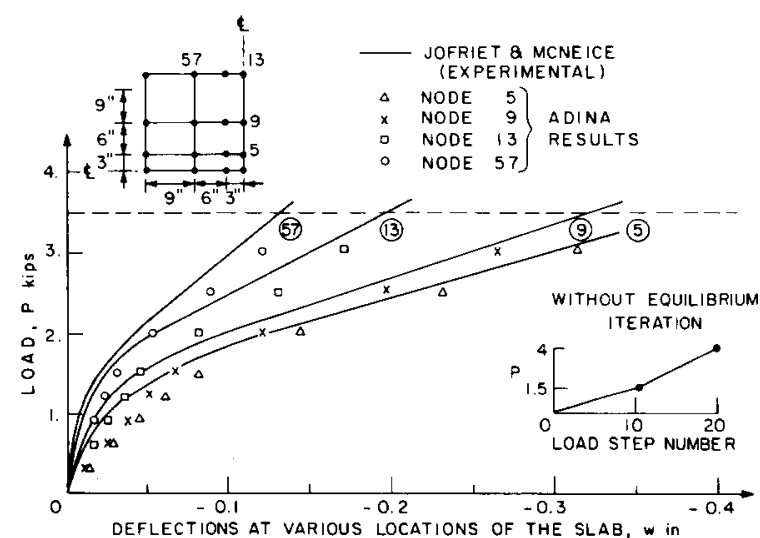

Fig. 21. Vertical deflections of the corner-supported concrete slab. 
model. Also, the weight of the plate was not included in the load application. Experimental and finite element solutions for this problem have been obtained by Jofriet and McNeice [25] and Lin and Scordelis [15], respectively.

Fig. 21 gives the loading procedure used in the ADINA analysis and compares displacements predicted in this study with the experimental results. Essentially, the same analysis results were also obtained with a total of 10 load steps. It is seen that the calculated and experimentally observed displacements at large load levels compare reasonably well. In the analysis, very large displacements were predicted at $p=3.5 \mathrm{kips}$ indicating collapse of the slab.

\section{Conclusions}

A finite element model for geometric and material nonlinear three-dimensional analysis of concrete and some rock structures has been presented. The model is based on a nonlinear uniaxial stress-strain relation that is generalized for two- and three-dimensional stress conditions. Tensile cracking and compression crushing, strain-softening and cyclic loading conditions are considered. The model has been implemented and some sample solutions have been presented.

When developing material nonlinear solution capabilities the two major difficulties encountered are that appropriate nonlinear material descriptions must be employed and that these descriptions must be numerically tractable, i.e., a stable and effective implementation must be possible. It is, of course, imperative that physically appropriate material descriptions be employed in a finite element formulation, but it is also important to operate on these descriptions in a stable, accurate and efficient manner, because otherwise the predicted response may be meaningless and can certainly not be interpreted with confidence. In the work reported in this paper, emphasis was placed on both of these aspects of material nonlinear finite element analysis.

The model proposed in this paper can already be employed effectively for the solution of various problems. The objective in the paper was to describe the basic model and some applications. However, it is realized that significant further studies, evaluations and improvements of the model are needed. For example, a detailed comparison of the model with other concrete and rock material models, the evaluation of the model in situations with significant strain softening effects, and the evaluation of the model to predict fracture discontinuities and strain localizations is important $[26,27]$. Such studies are currently being pursued and are expected to lead to further insight and improvements in the model.

\section{Acknowledgements}

The work reported in this paper has been supported financially by the ADINA users group. We would like to acknowledge gratefully this support. We also would like to thank J. Crawford of the Naval Civil Engineering Laboratory, Port Hueneme, for various valuable suggestions during the course of this work.

\section{References}

[1] O.C. Zienkiewicz, The Finite Element Method in Engineering Science (McGraw-Hill, New York, 1971).

[2] K.J. Bathe and E.L. Wilson, Numerical Methods in Finite Element Analysis (Prentice-Hall, 1976).

[3] R. Wegner, in: Formulations and Computational Algorithms in Finite Element Analysis, eds., K.J. Bathe, J.T. Oden and W. Wunderlich (MIT Press, Cambridge, 1977).

[4] L.P. Saenz, ACI J. 61 (1964) 1229.

[5] S. Popovics, ACI J. 67 (1970) 243.

[6] H. Kupfer, H.K. Hilsdorf and H. Rusch, ACI J. 66 (1969) 656. 
[7] T.C.Y. Liu, A.H. Nilson and F.O. Slate, ASCE J. Struct. Div. 98 (1972) 1025.

[8] M.H. Khan and B. Saugy, Concrete for Nuclear Reactors - ACI Publication SP-34 (1972).

[9] P. Launay and H. Gachon, Concrete for Nuclear Reactors, Vol. I - ACI Publication SP-34 (1972).

[10] O.C. Zienkiewicz, D.R.J. Owen, D.V. Phillips and G.C. Nayak, Nucl. Eng. Des. 20 (1972) 507.

[11] J.H. Argyris, G. Faust, J. Szimmat, E.P. Warnke and K.J. Willam, Nucl. Eng. Des. 28 (1974) 42.

[12] Z.P. Bažant, P. Bhat and C. Shieh, Northwestern University Structural Engineering Report 12-259 (1976).

[13] M. Suidan and W. Schnobrich, ASCE J. Struct. Div. 99 (1973) 2109.

[14] A.C. Scordelis, in: Proc. Spec. Conf. Finite Element Method in Civil Engineering, McGill University, Montreal (1972) p. 71.

[15] C.S. Lin and A.C. Scordelis, ASCE J. Struct. Div. 101 (1975) 523.

[16] Y. Sarne, Ph.D. Thesis, Massachusetts Institute of Technology (1974).

[17] K.J. Bathe, Massachusetts Institute of Technology Report 82448-1 (1977).

[18] J.C. Jaeger and N.G.W. Cook, Fundamentals of Rock Mechanics (Wiley, New York, 1976).

[19] K.J. Bathe, E. Ramm and E.L. Wilson, Int. Num. Methods Eng. 9 (1975) 353.

[20] K.J. Bathe, Massachusetts Institute of Technology Report 82448-2 (1977).

[21] K.J. Bathe, S. Bolourchi, S. Ramaswamy and M.D. Snyder, Nucl. Eng. Des. 46 (1978) 429.

[22] J.P. Laible, R.N. White and P. Gergely, Cornell University Department of Structural Engineering Report (1973).

[23] N.W. Krahl, N. Khachaturian and C.P. Siess, ASCE J. Struct. Div. 93 (1967) 235.

[24] M.A. Sozen and S.L. Paul. Nucl. Eng. Des. 8 (1968) 403.

[25] J.C. Jofriet and G.M. McNeice, ASCE J. Struct. Div. 97 (1971) 785.

[26] R.N. Murtha and J.E. Crawford, Naval Construction Battalion Center Technical Memorandum M-SI-78-6 (1977).

[27] M.P. Cleary, J. Pressure Vessel Petroleum Technol. 100 (1978) 2. 